\title{
Synchrotron self-Compton flaring of TeV blazars
}

\section{Linear electron cooling}

\author{
R. Schlickeiser and C. Röken \\ Institut für Theoretische Physik, Lehrstuhl IV: Weltraum- und Astrophysik, Ruhr-Universität Bochum, 44780 Bochum, Germany \\ e-mail: rsch@tp4.ruhr-uni-bochum.de
}

Received 11 September 2007 / Accepted 4 November 2007

\begin{abstract}
The vast improvement of the sensitivity of modern ground-based air Cherenkov telescopes, together with the sensitive flux measurements at lower frequencies, requires accurate elaborations of the theoretical radiation models for flaring blazars. Here the flaring of $\mathrm{TeV}$ blazars due to the synchrotron-self Compton (SSC) process is considered. We assume that, at the moment $t=t_{0}$, a flare in the emission knot occurs due to the instantaneous injection of monoenergetic $\left(E_{0}\right)$ ultrarelativistic electrons. The ultrarelativistic electrons are injected uniformly over the knot volume and at later times are subject to linear synchrotron radiation cooling in a magnetic field whose strength remains constant during the time evolution of the relativistic electrons.The generated synchrotron photons are subject to multiple Thomson-scattering off the cold electrons in the source giving rise to spatial photon diffusion. Optically thick and thin synchrotron radiation intensities and photon density distributions in the emission knot as functions of frequency and time are analytically determined. The synchrotron photons serve as target photons for the SSC process, which is calculated in the optically thin frequency range using the Thomson approximation of the inverse Compton cross section. It is shown that the optically thick part of the synchrotron radiation process provides a negligible contribution to the resulting SSC intensity at all frequencies and times.Because the high-energy $\mathrm{TeV}$ photons undergo no elastic multiple Compton scatterings, we neglect the influence of photon diffusion in the calculation of the SSC intensity and fluence distribution with energy. The SSC fluence exhibits a break at $E_{f}=15.8 b^{-1 / 3}$ $\mathrm{GeV}$ from a $\propto E_{s}^{-1 / 4}$-power law spectrum at lower photon energies $E_{t} \leq E_{s} \leq E_{f}$ to a $\propto E_{s}^{-2}\left[1-\left(E_{s} / E_{0}\right)^{7 / 3}\right]$-distribution at high energies $E_{f} \leq E_{s} \leq E_{0}$. The application to the observed TeV fluence spectrum of the flare of PKS 2155-304 on July 28, 2006 yields $\delta b^{-1 / 3}=27.1 \pm 6.5$. The emergent SSC light curve is independent of spatial photon diffusion and determined by the temporal variations on the relativistic electron density distribution and the synchrotron photon density. The comparison of the observed with the theoretical monochromatic synchrotron light curve determines the photon escape distribution.
\end{abstract}

Key words. radiation mechanisms: non-thermal - galaxies: active - gamma rays: theory

\section{Introduction}

During the last years the sensitivity of modern ground-based airCherenkov telescopes such as HESS, MAGIC and VERITAS, operating at photon energies above $0.1 \mathrm{TeV}$, has been vastly improved. For strong enough flaring blazar sources at cosmological distances such as PKS 2155-304 (redshift $z=0.116$ ) (Aharonian et al. 2007) and Mrk $421(z=0.030)$ (Woo et al. 2005; Albert et al. 2007), the observed high fluxes now allow the determination of accurate $\mathrm{TeV}$ photon spectra on time scales of minutes. It is anticipated that with the upcoming GLAST gammaray satellite mission similar sensitivities will soon be reached in the $\mathrm{MeV}-\mathrm{GeV}$ energy range. Combined with the available sensitive $\mathrm{X}$-ray and optical observations, these data probe the internal radiation mechanisms in BL Lac objects to unprecedented detail.

Clearly, the analysis of these data requires accurate elaborations of theoretical radiation models for flaring blazars. The combination of high observed luminosities with the observed short time variability in blazar flares indicates that the photon emission in blazars originates in relativistic jet knots that are beamed and Doppler-boosted towards the observer (e.g. Schlickeiser 1996). Superluminal expansions observed with VLBI (Piner \& Edwards 2004) provide evidence for moderate Doppler boosting factors in PKS 2155-304, although the
$\mathrm{TeV}$ variability time scales require the presence of much larger Doppler factors in these jets. After accounting for the Doppler time dilatation the intrinsic variability time scale $t_{\mathrm{var}}$ predominantly constrains the size $R$ of the emitting source. However, the intrinsic cooling time of the relativistic particles, generating the nonthermal photons in the source, then also has to be smaller than $R / c$. The cooling time of the radiating relativistic particles controls the time dependence of the nonthermal radiation intensity $I(v, t)$ in the jet knot. The time dependence of the spectrum of radiation emerging from the cloud is given by the product $\psi(v, t)=I(v, t) P(t)$ where $P(t)$ is the frequency-independent distribution of photons over their time of escape from the emission knot (Sunyaev \& Titarchuk 1980, 1985).

In the specific case of the flare of PKS 2155-304 on 2006 July 28 the quasi-simultaneous spectral energy distribution provided by the Swift data reported in Foschini et al. (2007) suggests that the main gamma-ray emission channel seems to be the synchrotron-self Compton process whereas synchrotron radiation provides the photons in the optical, UV and X-ray band. In this work we therefore study the flaring of TeV blazars due to the synchrotron-self Compton (SSC) process. Existing studies in the literature of the variability of the SSC-process (e.g. Mastichiadis \& Kirk 1997; Dermer et al. 1997; Chiaberge \& Ghisellini 1999; Sokolov et al. 2004; Böttcher 2007) rely mostly on numerical 
computational models for the combined time evolution of the emerging photons and the radiating particle energy distribution function injected at the rate $q_{0}$ with an initial power law distribution function between lower $\left(\gamma_{\min }\right)$ and upper $\left(\gamma_{\max }\right)$ cut-offs. Flares due to impulsive changes of $\gamma_{\max }, q_{0}$ and the magnetic field strength in the emitting source then have been examined. Our study here differs by its analytical approach, also allowing different to power law injections, by the inclusion of spatial photon diffusion due to multiple Compton scattering off the cold electron plasma in the emission knot, and by its emphasis on $\mathrm{TeV}$ light curves.

In our study we assume that at the moment $t=t_{0}$ a flare of the emission knot occurs due to the instantaneous injection of monoenergetic ultrarelativistic electrons. The ultrarelativistic electrons are injected uniformly over the knot volume. The emission knot itself moves with the relativistic bulk speed $V$ with respect to an observer. The emission knot is modeled as a spherical magnetised plasma cloud of radius $R$ which consists of cold fully ionized electrons and protons of uniform density $N_{e}$ and a magnetic field of constant strength $B$ which is randomly oriented on scales larger than the gyroradii of the injected ultrarelativistic electrons. The existence of this magnetic field is necessary for the SSC radiation and associated radiative cooling of the ultrarelativistic electrons. The optical depth of the knot with respect to Thomson scattering is $K_{0}=\sigma_{T} N_{e} R$. The Thomson scattering results in the spatial diffusion of photons in the emission knot after their generation.

The knot's magnetic field may be generated from the interaction of the relativistically moving knot with the surrounding ambient interstellar or intergalactic medium that also causes the injection of ultrarelativistic charged particles by the relativistic pick-up process (Pohl \& Schlickeiser 2000; Gerbig $\&$ Schlickeiser 2007; Stockem et al. 2007). This interaction is a prominent example of the relativistic collision of plasma shells with different properties (temperature, density, composition etc.). Experimentally (Kapetanakos 1974; Tatarakis et al. 2003) and from numerous particle-in-cell (PIC) simulations (e.g. Lee \& Lampe 1973; Nishikawa et al. 2003; Silva et al. 2003; Frederiksen et al. 2004; Sakai et al. 2004; Jaroschek et al. 2005; $\mathrm{Ng} \&$ Noble 2006) such collisions of plasma shells lead to the onset of linear Weibel-type plasma instabilities perpendicular to the flow directions in both unmagnetized and slightly magnetized plasmas. The PIC simulations of electron-proton and electron-positron plasmas demonstrate that these instabilities generate magnetic fields in the form of aperiodic fluctuations at almost equipartition ( $e_{B} \simeq 0.1$ typically for electron-proton plasmas) strength on the shortest plasma time scale. The aperiodic magnetic fluctuations will isotropise the initially anisotropic particle distributions in the rest frame of the jet leading to an efficient pick-up of nearly monoenergetic relativistic electrons and protons (Schlickeiser et al. 2002).

Although other (than monoenergetic) injection energy spectra of ultrarelativistic electrons and other (than instantaneous) injection time profiles are physically plausible, the case of monoenergetic instantaneous injection considered here is mathematically very convenient, as the solution of the time-dependent kinetic equation for the electrons for this case serves as Green's function for more complex injection energy spectra and time profiles, as long as the electron synchrotron cooling is linear, i.e. the magnetic field strength remains constant during the time evolution of the relativistic electrons (Schlickeiser \& Lerche 2007a). The solutions for more complex injection energy spectra and time profiles $Q(E, t)$ are then simply obtained by quadratures involving the Green's function solution calculated here.
Adopting monoenergetic injection distributions of ultrarelativistic electrons is also useful because the electron radiation losses quickly quench any broad injected energy distribution into a rather narrow energy distribution both in the linear (Kardashev 1962) and nonlinear (Schlickeiser \& Lerche 2007b) cooling cases.

The organization of the paper is as follows: in Sects. 2 and 3 we solve the time-dependent evolution of the volume-averaged relativistic electron population inside the radiating source under linear synchrotron cooling and provide the necessary intrinsic synchrotron radiation formulas, respectively. Intrinsic synchrotron radiation intensities are calculated in Sect. 4 . The emergent synchrotron radiation flux is given by the combination of the energy-independent photon escape time distribution, calculated in Appendix B, and by the energy-dependent synchrotron intensity. In Sect. 5 the optically thin synchrotron self-Compton emission is determined using the Thomson limit of the KleinNishina cross section. The multiwavelength intensity distribution from the combination of the synchrotron and SSC intensities is discussed in Sect. 6. By time-integration of the SSC intensity we derive in Sect. 7 the SSC fluence distribution. In Sect. 8 the light curves of the emerging synchrotron and SSC fluxes are calculated. Section 9 summarizes the main results of our investigation.

\section{Linear electron synchrotron cooling}

All physical quantities are calculated in a coordinate system comoving with the radiation source.

The competition between the instantaneous injection of ultrarelativistic electrons $\left(\gamma_{0} \gg 1\right)$ at the rate $Q(\gamma, t)=q_{0} \delta(\gamma-$ $\left.\gamma_{0}\right) \delta\left(t-t_{0}\right)$ at the time $t=t_{0}$ and the electron synchrotron energy losses implies for a time-dependent evolution of the volume-averaged relativistic electron population inside the radiating source (Kardashev 1962) given through

$$
\frac{\partial n(\gamma, t)}{\partial t}-\frac{\partial}{\partial \gamma}[|\dot{\gamma}| n(\gamma, t)]=q_{0} \delta\left(\gamma-\gamma_{0}\right) \delta\left(t-t_{0}\right)
$$

with the synchrotron energy loss rate

$|\dot{\gamma}|=D_{0} \gamma^{2}, D_{0}=\frac{4}{3} \frac{c \sigma_{T}}{m_{e} c^{2}} U_{B}=2.66 \cdot 10^{-14}\left[\frac{U_{B}}{m_{e} c^{2}}\right] \mathrm{s}^{-1}$

$n(\gamma, t)$ denotes the volume-averaged differential number density. Throughout we consider ultrarelativistic electrons $(\gamma \gg 1)$ so that the relation $p \simeq m_{e} c \gamma$ is appropriate, implying the relation $N(p, t)=n(\gamma, t) / m_{e} c$ between the respective differential electron densities.

In the case of linear cooling with constant energy density $U_{B}$ the rate (2) is constant, and the solution of the kinetic Eq. (1) is

$n_{L}\left(\gamma, \gamma_{0}, t, t_{0}\right)=q_{0} H\left[\gamma_{0}-\gamma\right] \delta\left(\gamma-\gamma_{L}(t)\right)$

where $H[x]$ denotes Heaviside's step function and

$\gamma_{L}(t)=\frac{\gamma_{0}}{1+D_{0} \gamma_{0}\left(t-t_{0}\right)}$.

\section{Intrinsic synchrotron radiation formula}

The pitch-angle averaged synchrotron power of a single electron in vacuum is (Crusius \& Schlickeiser 1986) $P(v, \gamma)=P_{0} v_{s} W(x)$ with

$x=\frac{v}{v_{s} \gamma^{2}}$ 
and $P_{0}=8.763 \times 10^{-29} \mathrm{erg} \mathrm{Hz}^{-1}$ and $v_{s}=3 e B /\left(4 \pi m_{e} c\right)$ being proportional to the electron gyrofrequency. The function $W(x)$ is well approximated by

$W(x) \simeq a_{0} x^{1 / 3} \mathrm{e}^{-x}$

where $a_{0}=1.151$.

In an homogeneous source of radius $R$ the synchrotron intensity from relativistic electrons with the volume-averaged differential density $n(\gamma, t)$ then is

$$
\begin{aligned}
I(v, t) & =\frac{j(v, t)}{\mu(v, t)}[1-\exp (-D(v, t))] \\
& \simeq \begin{cases}j(v, t) R & \text { for } D(v, t) \leq 1 \\
\frac{j(v, t)}{\mu(v, t)} & \text { for } D(v, t)>1\end{cases}
\end{aligned}
$$

in terms of the spontaneous synchrotron emission coefficient

$j(v, t)=\frac{1}{4 \pi} \int_{0}^{\infty} \mathrm{d} \gamma n(\gamma, t) P(v, \gamma)$,

the synchrotron absorption coefficient

$\mu(v, t)=\frac{1}{8 \pi m v^{2}} \int_{0}^{\infty} \mathrm{d} \gamma \frac{n(\gamma, t)}{\gamma^{2}} \frac{\mathrm{d}}{\mathrm{d} \gamma}\left[\gamma^{2} P(v, \gamma)\right]$,

and the synchrotron optical thickness

$D(v, t)=R \mu(v, t)$.

With the approximation (6) we immediately derive

$$
\begin{aligned}
j(v, t)= & \frac{a_{0} P_{0} v_{s}}{4 \pi}\left(\frac{v}{v_{s}}\right)^{1 / 3} \\
& \times \int_{0}^{\infty} \mathrm{d} \gamma n(\gamma, t) \gamma^{-2 / 3} \exp \left[-\frac{v}{v_{s} \gamma^{2}}\right]
\end{aligned}
$$

and

$$
\begin{aligned}
D(v, t)= & R \mu(v, t)=\frac{a_{0} P_{0} R}{8 \pi m v_{s}}\left(\frac{v}{v_{s}}\right)^{-5 / 3} \\
& \times \int_{0}^{\infty} \mathrm{d} \gamma \frac{n(\gamma, t)}{\gamma^{2}} \frac{\mathrm{d}}{\mathrm{d} \gamma}\left[\gamma^{4 / 3} \exp \left[-\frac{v}{v_{s} \gamma^{2}}\right]\right],
\end{aligned}
$$

respectively. Because

$$
\begin{aligned}
\frac{\mathrm{d}}{\mathrm{d} \gamma}\left[\gamma^{4 / 3} \exp \left[-\frac{v}{v_{s} \gamma^{2}}\right]\right]= \\
\quad \frac{4}{3} \gamma^{1 / 3} \exp \left[-\frac{v}{v_{s} \gamma^{2}}\right]\left[1+\frac{3 v}{2 v_{s} \gamma^{2}}\right]
\end{aligned}
$$

is always positive, the synchrotron optical depth and the absorption coefficient are also always positive, excluding the synchrotron maser case in vacuum. With the identity (13) we derive for Eq. (12)

$$
\begin{aligned}
D(v, t)= & \frac{a_{0} P_{0} R}{6 \pi m v_{s}}\left(\frac{v}{v_{s}}\right)^{-5 / 3} \\
& \times \int_{0}^{\infty} \mathrm{d} \gamma n(\gamma, t) \gamma^{-5 / 3}\left[1+\frac{3 v}{2 v_{s} \gamma^{2}}\right] \exp \left[-\frac{v}{v_{s} \gamma^{2}}\right] .
\end{aligned}
$$

\section{Synchrotron radiation intensities}

We define the normalised time scale

$\tau \equiv D_{0} \gamma_{0}\left(t-t_{0}\right)$

and insert the linear electron density distribution (3)

$n_{L}\left(\gamma, \gamma_{0}, \tau\right)=q_{0} H\left[\gamma_{0}-\gamma\right] \delta\left(\gamma-\frac{\gamma_{0}}{1+\tau}\right)$

in the synchrotron emissivities (11) and (14).

In terms of the normalised frequency

$\omega \equiv \frac{v}{v_{s} \gamma_{0}^{2}}$

we obtain after performing the $\gamma$-integration for the spontaneous emission coefficient (11)

$j(\omega, \tau)=\frac{a_{0} P_{0} q_{0} v_{s}}{4 \pi}\left[\omega(1+\tau)^{2}\right]^{1 / 3} \exp \left[-\omega(1+\tau)^{2}\right]$

and for the optical depth

$D(\omega, \tau)=\frac{a_{0} P_{0} q_{0} R}{6 \pi m v_{s} \gamma_{0}^{5}} \frac{(1+\tau)^{5 / 3}}{\omega^{5 / 3}}$

$$
\times\left[1+\frac{3}{2} \omega(1+\tau)^{2}\right] \exp \left[-\omega(1+\tau)^{2}\right]
$$

The frequency normalisation (17) refers to the initial characteristic synchrotron frequency

$v_{s} \gamma_{0}^{2}=4.2 \times 10^{20} b \gamma_{7}^{2} \mathrm{~Hz}$

in terms of characteristic values of the initial electron Lorentz factor $\gamma_{0}=10^{7} \gamma_{7}$ and magnetic field strength $B=1 b$ Gauss. The normalised frequency values $\omega$ are smaller than unity at later times.

It is convenient to define the characteristic frequency

$\omega_{1} \equiv\left[\frac{a_{0} P_{0} q_{0} R}{6 \pi v_{s} m \gamma_{0}^{5}}\right]^{3 / 5}=2.8 \times 10^{-14}\left(\frac{q_{5} R_{15}}{b}\right)^{3 / 5} \gamma_{7}^{-3}$

for characteristic values of the electron injection rate $q_{0}=10^{5} q_{5}$ electrons $\mathrm{cm}^{-3}$ and the source radius $R=10^{15} R_{15} \mathrm{~cm}$. In terms of the characteristic frequency (21) the optical depth (19) reads

$D(\omega, \tau)=\mu(\omega, \tau) R=\left(\frac{(1+\tau) \omega_{1}}{\omega}\right)^{5 / 3}$

$$
\times\left[1+\frac{3}{2} \omega(1+\tau)^{2}\right] \exp \left[-\omega(1+\tau)^{2}\right]
$$

The synchrotron optical depth and the optically thin and thick synchrotron intensities are considered in Appendix A. 


\subsection{Synchrotron intensity variation}

The intensity variation for fixed frequencies as a function of time has been already discussed for optically thin emission $\omega \gg \omega_{t}$ by Schlickeiser \& Lerche (2007a). The time-variation of the transition intensity is given in Eqs. (112)-(113). It remains to discuss the light curves at optically thick frequencies. From Eq. (106) we obtain

$$
\begin{gathered}
\frac{I\left(\omega \ll \omega_{t}, \tau\right)}{I_{0}} \frac{\omega_{1}^{5 / 3}}{\omega^{2}}=\left((1+\tau)\left[1+\frac{3}{2} \omega(1+\tau)^{2}\right]\right)^{-1} \\
\simeq \begin{cases}(1+\tau)^{-1} & \text { for } \tau \leq \omega_{1}^{-1 / 3} \\
\tau^{-1}\left[1+\frac{3}{2} \omega \tau^{2}\right]^{-1} & \text { for } \tau>\omega_{1}^{-1 / 3}\end{cases}
\end{gathered}
$$

At intermediate times $1<\tau \leq \omega_{1}^{-1 / 3}$ the optically thick intensity decreases inversely proportional to the normalised time, whereas at large times $\tau>\omega_{1}^{-1 / 3}$ it decreases $\propto \tau^{-2}$.

\subsection{Synchrotron photon density distribution}

In terms of the differential synchrotron photon number density $N(v, t)$ the synchrotron intensity is

$I(v, t)=\frac{c}{4 \pi} h v N(v, t)$.

In terms of the normalised photon energy

$\epsilon=\frac{h v}{m_{e} c^{2}}$

we derive

$N(\epsilon, t)=N(v, t) \frac{\mathrm{d} v}{\mathrm{~d} \epsilon}=\frac{4 \pi}{h c \epsilon} I\left(v=\frac{m_{e} c^{2} \epsilon}{h}, t\right)$.

Recalling from Eq. (17) that $v=v_{s} \gamma_{0}^{2} \omega$ we define

$\epsilon_{0}=\frac{h v_{s} \gamma_{0}^{2}}{m_{e} c^{2}}=3.4 b \gamma_{7}^{2}$

so that

$N(\epsilon, t)=\frac{4 \pi}{h c \epsilon} I\left(\omega=\frac{\epsilon}{\epsilon_{0}}, t\right)$.

With the synchrotron intensities (106) and (108) we obtain for optically thin energies $\epsilon>\epsilon_{t}=\epsilon_{0} \omega_{t}$

$N\left(\epsilon>\epsilon_{t}, \tau\right)=\frac{4 \pi I_{0}}{h c \epsilon_{0}}\left(\frac{\epsilon_{0}(1+\tau)}{\epsilon}\right)^{2 / 3} \exp \left[-\frac{\epsilon}{\epsilon_{0}}(1+\tau)^{2}\right]$

and at optically thick energies

$N\left(\epsilon \leq \epsilon_{t}, \tau\right)=\frac{4 \pi I_{0} \omega_{1}^{-5 / 3}}{h c \epsilon_{0}} \frac{\epsilon}{\epsilon_{0}(1+\tau)}\left[1+\frac{3 \epsilon}{2 \epsilon_{0}}(1+\tau)^{2}\right]^{-1}$.

\subsection{Emergent synchrotron intensity}

For the standard parameters adopted the energies of synchrotron photons are nonrelativistic, see Eq. (48) below. The time dependence of the spectrum of synchrotron radiation emerging from the emission knot is then given by the product

$\psi_{\mathrm{s}}(v, t)=I_{\mathrm{s}}(v, t) P(t)$, where $P(t)$ is the distribution of nonrelativistic photons over their time of escape from the emission knot (Sunyaev \& Titarchuk $1980,1985)$ and $I_{\mathrm{s}}(v, t)$ stands for the synchrotron intensity (Sect. 4.2) at the edge $R$ of the emission knot. The frequency independent photon escape time distribution is calculated in Appendix B.

For the case of multiple Compton scattering considered here, any photon escape distribution $P(t)$ does not depend on frequency or energy. Spatial photon transport alone therefore cannot explain the observed synchrotron light curves from blazars because multifrequency observations at optical and X-ray energies yield different time dependences in the different synchrotron energy bands. Clearly, a combination with the energy dependent relativistic electron light curves is necessary.

\section{Synchrotron self-Compton emission}

For the calculation of the synchrotron self-Compton emission we use the $\delta$-function approximation for inverse Compton scattering (Dermer \& Schlickeiser 1993)

$\dot{N}_{c}\left(\epsilon_{s}, \tau\right)=\frac{c \sigma_{T}}{3 \pi} \int_{0}^{\infty} \mathrm{d} \epsilon N(\epsilon, \tau) \int_{0}^{1 / \epsilon} \mathrm{d} \gamma n(\gamma, \tau) \delta\left(\epsilon_{s}-\gamma^{2} \epsilon\right)$

yielding the differential number density of Compton scattered synchrotron (SSC) photons. $\epsilon_{s}=E_{\gamma} / m_{e} c^{2}$ is the normalised scattered photon energy. The limit imposed on the $\gamma$-integral restricts the scattering to the Thomson regime.

Equation (32) yields for the corresponding spontaneous SSC emission coefficient

$j_{c}(v, t)=\frac{h \epsilon_{s}}{4 \pi} \dot{N}_{c}\left(\epsilon_{s}, \tau\right) \frac{\mathrm{erg}}{\mathrm{cm}^{3} \text { ster Hz s}}$.

Neglecting the effects due to stimulated SSC emission and absorption the SSC intensity then is

$I_{c}(v, t)=R j_{c}(v, t)=\frac{h R \epsilon_{s}}{4 \pi} \dot{N}_{c}\left(\epsilon_{s}, \tau\right) \frac{\text { erg }}{\mathrm{cm}^{2} \text { ster Hz s}}$.

We insert the relativistic electron distribution (3) in Eq. (32) to derive

$$
\begin{aligned}
\dot{N}_{c}\left(\epsilon_{s}, \tau\right) & =\frac{c \sigma_{T} q_{0}}{3 \pi} \int_{0}^{(1+\tau) / \gamma_{0}} \mathrm{~d} \epsilon N(\epsilon, \tau) \delta\left[\epsilon_{s}-\frac{\epsilon \gamma_{0}^{2}}{(1+\tau)^{2}}\right] \\
& =\frac{c \sigma_{T} q_{0}}{3 \pi} \frac{(1+\tau)^{2}}{\gamma_{0}^{2}} H\left[\frac{\gamma_{0}}{1+\tau}-\epsilon_{s}\right] N\left(\frac{(1+\tau)^{2} \epsilon_{s}}{\gamma_{0}^{2}}, \tau\right)
\end{aligned}
$$

Inserting this result in Eq. (34) yields

$$
\begin{aligned}
I_{c}\left(\epsilon_{s}, \tau\right)= & \frac{c \sigma_{T} h R q_{0}}{12 \pi^{2}} \frac{(1+\tau)^{2} \epsilon_{s}}{\gamma_{0}^{2}} \\
& \times H\left[\frac{\gamma_{0}}{1+\tau}-\epsilon_{s}\right] N\left(\frac{(1+\tau)^{2} \epsilon_{s}}{\gamma_{0}^{2}}, \tau\right) .
\end{aligned}
$$

Inserting the synchrotron photon number densities (29) and (30) we find

$$
\begin{gathered}
I_{c}\left(\epsilon_{s} \leq \frac{\epsilon_{t} \gamma_{0}^{2}}{(1+\tau)^{2}}, \tau\right)=\frac{\sigma_{T} R q_{0} I_{0}}{3 \pi \omega_{1}^{5 / 3}} H\left[\frac{\gamma_{0}}{1+\tau}-\epsilon_{s}\right] \\
\times \frac{\epsilon_{s}^{2}(1+\tau)^{3}}{\epsilon_{0}^{2} \gamma_{0}^{4}}\left[1+\frac{3}{2} \frac{\epsilon_{s}}{\epsilon_{0} \gamma_{0}^{2}}(1+\tau)^{4}\right]^{-1}
\end{gathered}
$$


and

$$
\begin{aligned}
I_{c}\left(\epsilon_{s}>\right. & \left.\frac{\epsilon_{t} \gamma_{0}^{2}}{(1+\tau)^{2}}, \tau\right)=\frac{\sigma_{T} R q_{0} I_{0}}{3 \pi} H\left[\frac{\gamma_{0}}{1+\tau}-\epsilon_{s}\right] \\
& \times\left(\frac{\epsilon_{s}}{\epsilon_{0} \gamma_{0}^{2}}\right)^{1 / 3}(1+\tau)^{4 / 3} \exp \left[-\frac{\epsilon_{s}(1+\tau)^{4}}{\epsilon_{0} \gamma_{0}^{2}}\right],
\end{aligned}
$$

respectively.

We now introduce practical units defining first using the earlier values (27) and (21):

$\epsilon_{k}(\tau)=\frac{\epsilon_{t}(\tau) \gamma_{0}^{2}}{(1+\tau)^{2}}=3.4 \times 10^{14} \frac{b \gamma_{7}^{4} \omega_{t}(\tau)}{(1+\tau)^{2}}$.

For $\tau$ smaller and larger than $\tau_{1}$, where

$\tau_{1}=\omega_{1}^{-1 / 3}=3.3 \times 10^{4} \frac{b^{1 / 5} \gamma_{7}}{q_{5}^{1 / 5} R_{15}^{1 / 5}}$,

Eq. (104) yields

$\epsilon_{k}\left(\tau<\tau_{1}\right)=\frac{\epsilon_{k}(0)}{1+\tau}, \quad \epsilon_{k}(0)=9.52 b^{2 / 5} R_{15}^{3 / 5} q_{5}^{3 / 5} \gamma_{7}$

and

$\epsilon_{k}\left(\tau \geq \tau_{1}\right)=\epsilon_{k}\left(\tau_{1}\right)\left(\frac{\tau}{\tau_{1}}\right)^{-4}\left[0.4+5 \ln \left(\tau / \tau_{1}\right)\right]$,

$\epsilon_{k}\left(\tau_{1}\right)=2.9 \times 10^{-4} b^{1 / 5} R_{15}^{4 / 5} q_{5}^{4 / 5}$,

respectively. The maximum SSC photon energy is strictly determined by the limit set by the Thomson regime

$\epsilon_{s, \max }=10^{7} \frac{\gamma_{7}}{1+\tau}$

and by the maximum value of the function determining the $\epsilon_{s}$-behaviour in Eq. (38):

$\epsilon_{m}=\frac{1}{3} \frac{\epsilon_{0} \gamma_{0}^{2}}{(1+\tau)^{4}}=1.13 \times 10^{14} \frac{b \gamma_{7}^{4}}{(1+\tau)^{4}}$.

The maximum SSC intensity occurs at

$$
\begin{aligned}
\epsilon_{s, m} & =\min \left[\epsilon_{s, \max }, \epsilon_{m}\right] \\
& \simeq\left\{\begin{array}{ll}
10^{7} \frac{\gamma_{7}}{1+\tau} & \text { for } \tau \leq \tau_{4}=224 b^{1 / 3} \gamma_{7} \\
1.13 \times 10^{14} \frac{b \gamma_{7}^{4}}{(1+\tau)^{4}} & \text { for } \tau>\tau_{4}
\end{array} .\right.
\end{aligned}
$$

In Fig. 1 we show the time variation of the photon energies $\epsilon_{k}$, $\epsilon_{s, \max }$ and $\epsilon_{m}$ for standard parameters:

(1) We first note that $\epsilon_{k}$, which reflects the energy of Compton scattered synchrotron photons whose frequency equals the synchrotron transition frequency $\omega_{t}$, is always decreasing with time: a different behaviour than $\omega_{t}$ itself (see Fig. 6).

(2) For all times the maximum scattered photon energy $\epsilon_{s, \max }$ is much larger than $\epsilon_{k}$, indicating that at all times the high energy SSC emission results from the scattering of synchrotron photons in the optically thin frequency range.

(3) For times smaller than 224 no exponential turn-over of the SSC emission will occur because the Thomson limit sets in before. Here the SSC intensity exhibits a spectral flattening at $\epsilon_{k}(\tau)$ from the $\propto \epsilon^{2}$-variation of Eq. (37) to the $\propto \epsilon^{1 / 3}$ variation of Eq. (38).

(4) For times larger than 224 the exponential turnover will be exhibited by the SSC intensity spectrum at high photon energies.

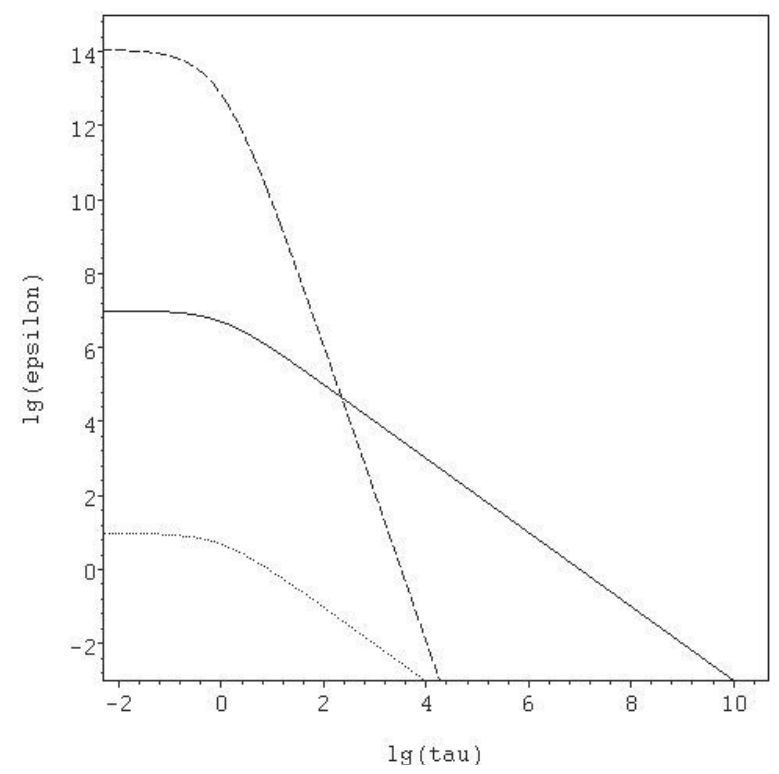

Fig. 1. Time variation of the photon energies $\epsilon_{k}$ (dotted curve), $\epsilon_{s, \max }$ (full curve) and $\epsilon_{m}$ (dashed curve) for standard parameters.

\section{Multiwavelength intensity spectrum}

In Fig. 2 we summarize the overall intensity distributions from the synchrotron and SSC radiation process as a function of the photon energy $E=\epsilon m_{e} c^{2}$. Synchrotron radiation is optically thick below the transition energy $E_{t}(\tau)$, and optically thin above, extending up to the maximum synchrotron energy $E_{s, \max }(\tau)$. Likewise, the SSC intensity distribution exhibits a break at the energy $E_{D}(\tau)$ : at energies below $E_{D}$ photons from the optically thick part of the synchrotron spectrum are upscattered by the SSC processes, whereas at energies above $E_{D}$ photons from the optically thin part of the synchrotron spectrum are upscattered. The maximum SSC photon energy $E_{c, \max }(\tau)$ is determined either, by the maximum energy of the relativistic electrons for times less than $\tau \leq \tau_{3}=3.3 \times 10^{4} \gamma_{7}^{1 / 5}\left(R_{15} q_{5}\right)^{-1 / 5}$, or by the exponential roll-over at later times $\tau>\tau_{3}$. All these four characteristic photon energies depend on the time scale $\tau$ due to cooling of the relativistic electrons with time. In Fig. 2 we also indicate the variation with time $\tau$ of the four characteristic energies. We additionally note the variation with time of the maximum synchrotron $\left(I_{S, \max }(\tau)\right)$ and $\operatorname{SSC}\left(I_{S S C \text {, max }}(\tau)\right)$ intensities. Whereas the former is independent of time, the maximum SSC intensity $\left(I_{S S C, \max }(\tau) \propto 1+\tau\right)$ first increases with time for $\tau \leq \tau_{4}=224 b^{1 / 3} \gamma_{7}$, and attains a constant value at later times $\tau>\tau_{4}$

For completeness, we note the initial values (at time $\tau=0$ ) of the characteristic photon energies and maximum intensities and their scaling with physical parameters:

$E_{t}(0)=4.9 \times 10^{-8} b^{2 / 5}\left(R_{15} q_{5}\right)^{3 / 5} \gamma_{7}^{-1} \mathrm{eV}$,

$E_{D}(0)=\gamma_{0}^{2} E_{t}(0)=4.9 \times 10^{6} b^{2 / 5}\left(R_{15} q_{5}\right)^{3 / 5} \gamma_{7} \quad \mathrm{eV}$

$E_{s, \max }(0)=5.8 \times 10^{5} b \gamma_{7}^{2} \mathrm{eV}$,

$E_{c, \max }(0)=5.11 \times 10^{12} \gamma_{7} \mathrm{eV}$

$I_{S S C, \max }(0)=2.2 \times 10^{-8} R_{15} q_{5} b^{-1 / 3} \gamma_{7}^{4 / 3} I_{s, \max }(0)$. 


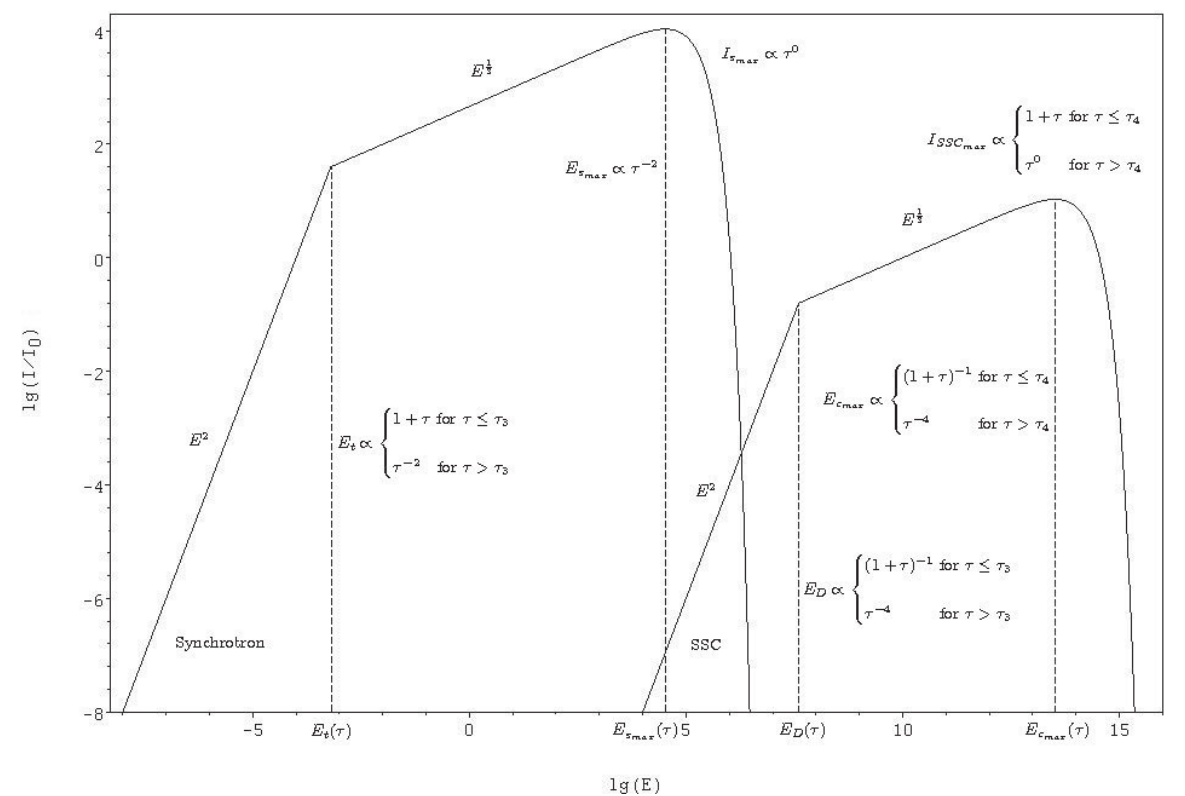

Fig. 2. Overall intensity distribution resulting from the synchrotron and SSC radiation processes. The scaling with time of the maximum intensities and the different characteristic radiation energies are indicated.

\section{Synchrotron self-Compton fluences}

In the relativistic photon energy regime $\epsilon \geq 1$ the large energy shift in a single Compton scattering removes a photon of energy $\epsilon$ from $I(\epsilon, t)$ upon scattering. Thus at these energies a diffusive (both spatial and in energy space) buildup of photons in the source interior cannot occur (Fabian et al. 1986; Lightman \& Zdziarski 1987). A photon either scatters and appears at a much lower $\epsilon$, or it escapes in a time $R / c$ from the source. Because the light travel time $R / c$ is longer than the short radiative electron loss times the emergent SSC radiation flux is given by $\psi(v, t)=I(v, t)$. The time-integrated SSC intensity yields the SSC fluence distribution

$F\left(\epsilon_{s}\right)=\int_{t_{0}}^{\infty} \mathrm{d} t I\left(\epsilon_{s}, t\right)=\frac{1}{D_{0} \gamma_{0}} \int_{0}^{\infty} \mathrm{d} \tau I\left(\epsilon_{s}, \tau\right)$.

We have to discuss two ranges of scattered photon energies: for energies $\epsilon_{s}>\epsilon_{k}(0)$ only the optically thin synchrotron photon distribution (38) contributes whereas at smaller energies $\epsilon_{s} \leq \epsilon_{k}(0)$ both, optically thin and thick, parts of the synchrotron photon distribution have to be taken into account. We consider each case in turn.

\subsection{Large scattered photon energies $\epsilon_{s}>\epsilon_{k}(0)$}

For energies $\epsilon_{s}>\epsilon_{k}(0)$ only Eq. (38) contributes, so that

$$
\begin{aligned}
F\left(\epsilon_{s}>\epsilon_{k}(0)\right) & =\frac{R \sigma_{T} q_{0} I_{0}}{3 \pi D_{0} \gamma_{0}^{5 / 3}}\left(\frac{\epsilon_{s}}{\epsilon_{0}}\right)^{1 / 3} \\
\times & \int_{0}^{\infty} \mathrm{d} \tau(1+\tau)^{4 / 3} H\left[\frac{10^{7} \gamma_{7}}{1+\tau}-\epsilon_{s}\right] \exp \left[-\frac{\epsilon_{s}(1+\tau)^{4}}{\epsilon_{0} \gamma_{0}^{2}}\right] \\
= & \frac{R \sigma_{T} q_{0} I_{0}}{3 \pi D_{0} \gamma_{0}^{5 / 3}}\left(\frac{\epsilon_{s}}{\epsilon_{0}}\right)^{1 / 3} \int_{1}^{\gamma_{0} / \epsilon_{s}} \mathrm{~d} z z^{4 / 3} \exp \left[-\frac{\epsilon_{s} z^{4}}{\epsilon_{0} \gamma_{0}^{2}}\right] .
\end{aligned}
$$

Substituting $y=\epsilon_{s} z / \gamma_{0}$ yields

$$
F\left(\epsilon_{s}>\epsilon_{k}(0)\right)=4 F_{0} \frac{\gamma_{0}^{2 / 3}}{\epsilon_{0}^{1 / 3} \epsilon_{s}^{2}} \int_{\epsilon_{s} / \gamma_{0}}^{1} \mathrm{~d} y y^{4 / 3} \exp \left[-\frac{\gamma_{0}^{2} y^{4}}{\epsilon_{0} \epsilon_{s}^{3}}\right]
$$

where

$F_{0}=\frac{R \sigma_{T} q_{0} I_{0}}{12 \pi D_{0}}$

The substitution $s=y^{4}$ yields for the fluence (53)

$$
\begin{aligned}
F\left(\epsilon_{s}>\epsilon_{k}(0)\right) & =F_{0} \frac{\gamma_{0}^{2 / 3}}{\epsilon_{0}^{1 / 3} \epsilon_{s}^{2}} \int_{\left(\epsilon_{s} / \gamma_{0}\right)^{4}}^{1} \mathrm{~d} s s^{-5 / 12} \exp \left[-\frac{\gamma_{0}^{2}}{\epsilon_{0} \epsilon_{s}^{3}} s\right] \\
& =F_{0} \frac{\epsilon_{f}}{\epsilon_{s}^{2}} \int_{\left(\epsilon_{s} / \gamma_{0}\right)^{4}}^{1} \mathrm{~d} s s^{-5 / 12} \exp \left[-\left(\frac{\epsilon_{f}}{\epsilon_{s}}\right)^{3} s\right]
\end{aligned}
$$

where we introduce

$\epsilon_{f}=\frac{\gamma_{0}^{2 / 3}}{\epsilon_{0}^{1 / 3}}=3.1 \times 10^{4} b^{-1 / 3}$.

For large scattered photon energies $\epsilon_{f} \ll \epsilon_{s} \leq \gamma_{0}$ the argument of the exponential function in the integral of Eq. (55) is very small for all values of $s$ yielding approximately

$F\left(\epsilon_{k}(0)<\epsilon_{f}<\epsilon s\right) \simeq \frac{12}{7} F_{0} \frac{\epsilon_{f}}{\epsilon_{s}^{2}}\left[1-\left(\frac{\epsilon}{\gamma_{0}}\right)^{7 / 3}\right]$.

For scattered photon energies $\epsilon_{k}(0) \leq \epsilon_{s}<\epsilon_{f}$ we substitute $w=$ $\left(\epsilon_{f} / \epsilon_{s}\right)^{3} s$ to obtain for the fluence (55)

$$
\begin{gathered}
F\left(\epsilon_{k}(0)<\epsilon_{s} \leq \epsilon_{f}\right)=\frac{F_{0}}{\epsilon_{f}^{3 / 4} \epsilon_{s}^{1 / 4}} \int_{\epsilon_{s} / \epsilon_{0} \gamma_{0}^{2}}^{\left(\epsilon_{f} / \epsilon_{s}\right)^{3}} \mathrm{~d} w w^{-5 / 12} \mathrm{e}^{-w} \\
=\frac{F_{0}}{\epsilon_{f}^{3 / 4} \epsilon_{s}^{1 / 4}}\left[\Gamma\left(\frac{7}{12}, \frac{\epsilon_{s}}{\epsilon_{0} \gamma_{0}^{2}}\right)-\Gamma\left(\frac{7}{12},\left(\epsilon_{f} / \epsilon_{s}\right)^{3}\right)\right]
\end{gathered}
$$

in terms of the incomplete gamma function $\Gamma(a, x)$.

The argument of the first incomplete gamma function $\epsilon_{S} /\left(\epsilon_{0} \gamma_{0}^{2}\right)$ is much smaller than unity for all scattered photon energies $\epsilon_{k}(0)<\epsilon_{s} \leq \epsilon_{f}$, so that we use here the approximation

$\Gamma(a, x \ll 1) \simeq \Gamma(a)-\frac{12}{7} x^{7 / 12}$. 
The argument of the second incomplete gamma function $\left(\epsilon_{f} / \epsilon_{s}\right)^{3}$ is much larger than unity for $\epsilon_{s} \ll \epsilon_{f}$, so that we use the asymptotic expansion of the incomplete gamma function for large arguments

$\Gamma(a, x \gg 1) \simeq x^{a-1} \mathrm{e}^{-x}$.

Collecting terms we obtain for the fluence (58)

$$
\begin{aligned}
F\left(\epsilon_{k}(0)<\epsilon_{s} \leq \epsilon_{f}\right) & \simeq \frac{F_{0}}{\epsilon_{f}^{3 / 4} \epsilon_{s}^{1 / 4}}\left[\Gamma\left(\frac{7}{12}\right) \frac{12}{7}\left(\epsilon_{s} / \epsilon_{0} \gamma_{0}^{2}\right)^{7 / 12}\right. \\
\left.-\left(\epsilon_{s} / \epsilon_{f}\right)^{5 / 4} \exp \left[-\left(\epsilon_{f} / \epsilon_{s}\right)^{3}\right]\right] & \simeq \Gamma\left(\frac{7}{12}\right) F_{0} \epsilon_{f}^{-3 / 4} \epsilon_{s}^{-1 / 4} .
\end{aligned}
$$

The spectral fluence above $\epsilon_{k}(0)=9.52$ exhibits a spectral steepening at $\epsilon_{f}$ from the $\propto \epsilon_{s}^{-1 / 4}$-power law below $\epsilon_{f}$ to the $\propto \epsilon_{s}^{-2}$ power law above $\epsilon_{f}$.

\subsection{Small scattered photon energies $\epsilon_{s} \leq \epsilon_{k}(0)$}

We now consider the spectral fluence at scattered photon energies below $\epsilon_{k}(0)=9.52$. Here both Eqs. (37) and (38) contribute. Starting with the scattered photon range $\epsilon_{k}\left(\tau_{1}\right) \leq \epsilon_{s} \leq \epsilon_{k}(0)$ we derive

$$
\begin{aligned}
& F\left(\epsilon_{k}\left(\tau_{1}\right) \leq \epsilon_{s} \leq \epsilon_{k}(0)\right)= \\
& \frac{1}{D_{0} \gamma_{0}}\left[\int_{0}^{\infty} \mathrm{d} \tau I\left(\epsilon_{s} \leq \epsilon_{k}(\tau)\right) H\left[\frac{\epsilon_{k}(0)}{1+\tau}-\epsilon_{s}\right]\right. \\
& \left.\quad+\int_{0}^{\infty} \mathrm{d} \tau I\left(\epsilon_{s}>\epsilon_{k}(\tau)\right) H\left[\epsilon_{s}-\frac{\epsilon_{k}(0)}{1+\tau}\right]\right] \\
& =\frac{1}{D_{0} \gamma_{0}}\left[\int_{0}^{\frac{\epsilon_{k}(0)}{\epsilon_{s}}-1} \mathrm{~d} \tau I\left(\epsilon_{s} \leq \epsilon_{k}(\tau)\right)\right. \\
& \left.\quad+\int_{\frac{\epsilon_{k}(0)}{\epsilon_{s}}-1}^{\infty} \mathrm{d} \tau I\left(\epsilon_{s}>\epsilon_{k}(\tau)\right)\right] .
\end{aligned}
$$

Inserting Eqs. (37) and (38) and substituting as before we find

$$
\begin{aligned}
& F\left(\epsilon_{k}\left(\tau_{1}\right) \leq \epsilon_{s} \leq \epsilon_{k}(0)\right)= \\
& 4 F_{0}\left[\frac{\omega_{1}^{-5 / 3} \epsilon_{s}^{2}}{\epsilon_{0}^{2} \gamma_{0}^{5}} \int_{1}^{\frac{\epsilon_{k}(0)}{\epsilon_{s}}} \mathrm{~d} z \frac{z^{3}}{1+\frac{3}{2} \frac{\epsilon_{s}}{\epsilon_{0} \gamma_{0}^{2}} z^{4}} H\left[\frac{\gamma_{0}}{z}-\epsilon_{s}\right]\right. \\
& \left.+\frac{\epsilon_{s}^{1 / 3}}{\epsilon_{0}^{1 / 3} \gamma_{0}^{5 / 3}} \int_{\epsilon_{k}(0) / \epsilon_{s}}^{\infty} \mathrm{d} z z^{4 / 3} \exp \left[-\frac{\epsilon_{s} z^{4}}{\epsilon_{0} \gamma_{0}^{2}}\right] H\left[\frac{\gamma_{0}}{z}-\epsilon_{s}\right]\right] \\
& =4 F_{0}\left[\frac{\omega_{1}^{-5 / 3} \epsilon_{s}^{2}}{\epsilon_{0}^{2} \gamma_{0}^{5}} \int_{1}^{\frac{\epsilon_{k}(0)}{\epsilon_{s}}} \mathrm{~d} z \frac{z^{3}}{1+\frac{3}{2} \frac{\epsilon_{s}}{\epsilon_{0} \gamma_{0}^{2}} z^{4}} H\left[\frac{\gamma_{0}}{z}-\epsilon_{s}\right]\right. \\
& \left.+\frac{\epsilon_{s}^{1 / 3}}{\epsilon_{0}^{1 / 3} \gamma_{0}^{5 / 3}} \int_{\epsilon_{k}(0) / \epsilon_{s}}^{\gamma_{0} / \epsilon_{s}} \mathrm{~d} z z^{4 / 3} \exp \left[-\frac{\epsilon_{s} z^{4}}{\epsilon_{0} \gamma_{0}^{2}}\right]\right]
\end{aligned}
$$

The first integral is readily solved so that

$F\left(\epsilon_{k}\left(\tau_{1}\right) \leq \epsilon_{s} \leq \epsilon_{k}(0)\right)=$

$$
\begin{aligned}
& F_{0}\left[\frac{\epsilon_{s}^{2}}{\epsilon_{0}^{2} \gamma_{0}^{5} \omega_{1}^{5 / 3}} \ln \frac{1+\frac{3}{2} \frac{\epsilon_{s}}{\epsilon_{0} \gamma_{0}^{2}}\left(\frac{\epsilon_{k}(0)}{\epsilon_{s}}\right)^{4}}{1+\frac{3}{2} \frac{\epsilon_{s}}{\epsilon_{0} \gamma_{0}^{2}}}\right. \\
& \left.+\frac{\gamma_{0}^{2 / 3}}{\epsilon_{0}^{1 / 3} \epsilon_{s}^{2}} \int_{\left(\epsilon_{k}(0) / \gamma_{0}\right)^{4}}^{1} \mathrm{~d} s s^{-5 / 12} \exp \left[-\frac{\gamma_{0}^{2}}{\epsilon_{0} \epsilon_{s}^{3}} s\right]\right] \\
& =F_{0}\left[3.87 \times 10^{-13} b q_{5}^{-1} R_{15}^{-1}\left(\frac{\epsilon_{s}}{\epsilon_{0}}\right)^{2} \ln \frac{1+\frac{3}{2} \frac{\epsilon_{s}}{\epsilon_{0} \gamma_{0}^{2}}\left(\frac{\epsilon_{k}(0)}{\epsilon_{s}}\right)^{4}}{1+\frac{3}{2} \frac{\epsilon_{s}}{\epsilon_{0} \gamma_{0}^{2}}}\right. \\
& \left.+\frac{\epsilon_{f}}{\epsilon_{s}^{2}} \int_{\left(\epsilon_{k}(0) / \gamma_{0}\right)^{4}}^{1} \mathrm{~d} s s^{-5 / 12} \exp \left[-\left(\frac{\epsilon_{f}}{\epsilon_{s}}\right)^{3} s\right]\right] .
\end{aligned}
$$

As before the second integral can be expressed in terms of two incomplete gamma functions yielding

$$
\begin{aligned}
& F\left(\epsilon_{k}\left(\tau_{1}\right) \leq \epsilon_{s} \leq \epsilon_{k}(0)\right)= \\
& F_{0}\left[3.87 \times 10^{-13} b q_{5}^{-1} R_{15}^{-1}\left(\frac{\epsilon_{s}}{\epsilon_{0}}\right)^{2} \ln \frac{1+\frac{3}{2} \frac{\epsilon_{s}}{\epsilon_{0} \gamma_{0}^{2}}\left(\frac{\epsilon_{k}(0)}{\epsilon_{s}}\right)^{4}}{1+\frac{3}{2} \frac{\epsilon_{s}}{\epsilon_{0} \gamma_{0}^{2}}}\right. \\
& \left.+\frac{1}{\epsilon_{f}^{3 / 4} \epsilon_{s}^{1 / 4}}\left(\Gamma\left(\frac{7}{12},\left(\frac{\epsilon_{f}}{\epsilon_{s}}\right)^{3}\left(\frac{\epsilon_{k}(0)}{\gamma_{0}}\right)^{4}\right)-\Gamma\left(\frac{7}{12},\left(\frac{\epsilon_{f}}{\epsilon_{s}}\right)^{3}\right)\right)\right] .
\end{aligned}
$$

The first logarithmic contribution to the fluence, representing synchrotron photons from the optically thick part of the spectrum, is negligibly small compared to the second contribution, representing synchrotron photons from the optically thin part of the synchrotron spectrum. With $\epsilon_{s} \ll \epsilon_{f}$ we derive

$F\left(\epsilon_{k}\left(\tau_{1}\right) \leq \epsilon_{s} \leq \epsilon_{k}(0)\right) \simeq \frac{F_{0}}{\epsilon_{f}^{3 / 4} \epsilon_{s}^{1 / 4}} \Gamma\left(\frac{7}{12},\left(\frac{\epsilon_{s, 1}}{\epsilon_{s}}\right)^{3}\right)$

with

$\epsilon_{s, 1}=\epsilon_{f}\left(\frac{\epsilon_{k}(0)}{\gamma_{0}}\right)^{4 / 3}=2.9 \times 10^{-4} b^{1 / 3} R_{15}^{4 / 5} q_{5}^{4 / 5}$

Therefore in the whole range $\epsilon_{k}\left(\tau_{1}\right) \leq \epsilon_{s} \leq \epsilon_{k}(0)$ we find that the argument of the incomplete gamma function is small compared to unity yielding

$F\left(\epsilon_{k}\left(\tau_{1}\right) \leq \epsilon_{s} \leq \epsilon_{k}(0)\right) \simeq \Gamma\left(\frac{7}{12}\right) \frac{F_{0}}{\epsilon_{f}^{3 / 4} \epsilon_{s}^{1 / 4}}$

identical to the earlier result (59). The $\propto \epsilon_{s}^{-1 / 4}$-power law fluence distribution holds for all energies between $\epsilon_{k}\left(\tau_{1}\right)$ and $\epsilon_{f}$. 
Now for scattered photon energies below $\epsilon_{s} \leq \epsilon_{k}\left(\tau_{1}\right)$ we find

$$
\begin{aligned}
& F\left(\epsilon_{s}<\epsilon_{k}\left(\tau_{1}\right)\right)=\frac{1}{D_{0} \gamma_{0}}\left[\int_{0}^{\infty} \mathrm{d} \tau I\left(\epsilon_{s} \leq \epsilon_{k}(\tau)\right) H\left[\frac{\epsilon_{k}\left(\tau_{1}\right) \tau_{1}^{4}}{\tau^{4}}-\epsilon_{s}\right]\right. \\
& \left.+\int_{0}^{\infty} \mathrm{d} \tau I\left(\epsilon_{s}>\epsilon_{k}(\tau)\right) H\left[\epsilon_{s}-\frac{\left.\epsilon_{k}\left(\tau_{1}\right) \tau_{1}^{4}\right)}{\tau^{4}}\right]\right] \\
& =\frac{1}{D_{0} \gamma_{0}}\left[\int_{0}^{\tau_{1}\left(\frac{\epsilon_{k}\left(\tau_{1}\right)}{\epsilon_{s}}\right)^{1 / 4}} \mathrm{~d} \tau I\left(\epsilon_{s} \leq \epsilon_{k}(\tau)\right)\right. \\
& \left.+\int_{\tau_{1}\left(\frac{\epsilon_{k}\left(\tau_{1}\right)}{\epsilon_{s}}\right)^{1 / 4}}^{\infty} \mathrm{d} \tau I\left(\epsilon_{s}>\epsilon_{k}(\tau)\right)\right] .
\end{aligned}
$$

Substituting as before we derive for $\tau_{1} \gg 1$

$$
\begin{aligned}
& F\left(\epsilon_{s}<\epsilon_{k}\left(\tau_{1}\right)\right)= \\
& \quad F_{0}\left[3.87 \times 10^{-13} b q_{5}^{-1} R_{15}^{-1}\left(\frac{\epsilon_{s}}{\epsilon_{0}}\right)^{2} \ln \frac{1+\frac{3}{2} \frac{\epsilon_{k}\left(\tau_{1}\right) \tau_{1}^{4}}{\epsilon_{0} \gamma_{0}^{2}}}{1+\frac{3}{2} \frac{\epsilon_{s}}{\epsilon_{0} \gamma_{0}^{2}}}\right. \\
& \left.+\frac{\epsilon_{f}}{\epsilon_{s}^{2}} \int_{\left(\epsilon_{s}^{3} \epsilon_{k}\left(\tau_{1}\right) \tau_{1}^{4} / \gamma_{0}^{4}\right)}^{1} \mathrm{~d} s s^{-5 / 12} \exp \left[-\left(\frac{\epsilon_{f}}{\epsilon_{s}}\right)^{3} s\right]\right] .
\end{aligned}
$$

In terms of incomplete gamma functions we obtain

$$
\begin{aligned}
& F\left(\epsilon_{s}<\epsilon_{k}\left(\tau_{1}\right)\right)= \\
& 3.87 \times 10^{-13} b q_{5}^{-1} R_{15}^{-1} F_{0}\left(\frac{\epsilon_{s}}{\epsilon_{0}}\right)^{2} \ln \frac{1+\frac{3}{2} \frac{\epsilon_{k}\left(\tau_{1}\right) \tau_{1}^{4}}{\epsilon_{0} \gamma_{0}^{2}}}{1+\frac{3}{2} \frac{\epsilon_{s}}{\epsilon_{0} \gamma_{0}^{2}}} \\
& \quad+\frac{F_{0}}{\epsilon_{f}^{3 / 4} \epsilon_{s}^{1 / 4}}\left(\Gamma\left[\frac{7}{12}, \frac{\epsilon_{f}^{3} \epsilon_{k}\left(\tau_{1}\right) \tau_{1}^{4}}{\gamma_{0}^{4}}\right]-\Gamma\left[\frac{7}{12},\left(\frac{\epsilon_{f}}{\epsilon_{s}}\right)^{3}\right]\right) .
\end{aligned}
$$

The argument of the second incomplete gamma function is large compared to unity while the argument of the second incomplete gamma function is a universal constant

$$
\frac{\epsilon_{f}^{3} \epsilon_{k}\left(\tau_{1}\right) \tau_{1}^{4}}{\gamma_{0}^{4}}=1.024
$$

yielding

$$
\begin{gathered}
F\left(\epsilon_{s}<\epsilon_{k}\left(\tau_{1}\right)\right)=F_{0}\left[3.87 \times 10^{-13} b q_{5}^{-1} R_{15}^{-1}\left(\frac{\epsilon_{s}}{\epsilon_{0}}\right)^{2}\right. \\
\left.\quad \times \ln \frac{2.513}{1+\frac{3}{2} \frac{\epsilon_{s}}{\epsilon_{0} \gamma_{0}^{2}}}+\frac{\Gamma\left[\frac{7}{12}, 1.024\right]}{\epsilon_{f}^{3 / 4} \epsilon_{s}^{1 / 4}}\right] .
\end{gathered}
$$

Again the contribution from the optically thin part dominates the logarithmic term for all values of $\epsilon_{s}$ demonstrating that the $\propto \epsilon_{s}^{-1 / 4}$-fluence distribution also holds in this scattered photon energy range

$$
F\left(\epsilon_{s}<\epsilon_{k}\left(\tau_{1}\right)\right) \simeq F_{0} \Gamma\left[\frac{7}{12}, 1.024\right] \epsilon_{f}^{-3 / 4} \epsilon_{s}^{-1 / 4} .
$$

This flat power law fluence distribution holds down to energies $\epsilon_{t}=\epsilon_{0} \omega_{t}$ below which the optically thickness of the source is provided by synchrotron radiation.

Summarizing this section: the SSC fluence without spatial photon diffusion exhibits a break at $E_{f}=15.8 b^{-1 / 3} \mathrm{GeV}$ from a $\propto E_{s}^{-1 / 4}$-power law spectrum at lower photon energies $E_{t} \leq$ $E_{s} \leq E_{f}$ to a $\propto E_{s}^{-2}\left[1-\left(E_{s} / E_{0}\right)^{7 / 3}\right]$-distribution at large energies $E_{f} \leq E_{s} \leq E_{0}$. Obviously, the identification of this break energy in the observed fluence distribution from flaring blazars will thus provide diagnostics for the product $\delta b^{-1 / 3}$ of the Doppler factor $\delta$ of the emission knot and the magnetic field strength $b$ in the source.

The HESS collaboration (Aharonian et al. 2007) reported for the TeV fluence of the flare of PKS 2155-304 on July 28, 2006 a transition from a $\left(E_{s}^{*}\right)^{-s_{1}}$ - to a $\left(E_{s}^{*}\right)^{-s_{2}}$ power law at $E_{f}^{*}$ with $s_{1}=1.71 \pm 0.06 \pm 0.10, s_{2}=2.53 \pm 0.05 \pm 0.10$ at $E_{f}^{*}=430 \pm$ $22 \pm 80 \mathrm{GeV}$. With $E_{f}^{*}=\delta E_{f}$ we derive for the product $\delta b^{-1 / 3}=$ $27.1 \pm 6.5$.

\section{Synchrotron and SSC light curves}

In this section we calculate the emerging photon intensities which in the case of synchrotron radiation include the effects of the photon escape distribution. Besides results valid for any photon escape distribution $P(t)$ we will also consider the special escape distributions (135) and (140) which were calculated for a uniform photon source distribution over the emission knot.

In terms of the normalised time scale (15) the scattering time $u$ reads

$u=\frac{c N_{e} \sigma_{T}}{D_{0} \gamma_{0}} \tau=\frac{3 m_{e} c^{2} N_{e}}{U_{B} \gamma_{0}} \tau=1.543 \times 10^{-2} \frac{N_{e, 10}}{b^{2} \gamma_{7}} \tau$.

The time $u=K_{0}^{2}$ then corresponds to $\tau=\tau_{2}$ with

$\tau_{2}=2.87 \times 10^{3} N_{e, 10} R_{15}^{2} b^{2} \gamma_{7}$

The two special escape distribitions (135) and (140) then read

$P_{1}\left(\tau \leq \tau_{2}\right)=0.226 N_{e, 10} R_{15}[1+\alpha \tau]^{-1 / 2}$

and

$P_{2}\left(\tau \geq \tau_{2}\right)=P_{0} \exp [-\beta \tau]$,

respectively, with

$\alpha=3.64 \times 10^{-2} \frac{N_{e, 10}}{b^{2} \gamma_{7}}, \beta=1.15 \times 10^{-3} b^{-2} \gamma_{7}^{-1} N_{e, 10}^{-1} R_{15}^{-2}$,

$P_{0}=\frac{0.045}{N_{e, 10}^{2} R_{15}^{2}}$.

The emergent synchrotron photon intensity then is given by

$\Psi_{s}(v, \tau)=I_{s}(v, \tau) P(\tau)$.

Using observed synchrotron photon intensities for $\psi_{s}(\nu, \tau)$ we may infer the synchrotron photon escape time distribution from the ratio

$P(\tau)=\psi_{\mathrm{s}, \mathrm{obs}} / I_{S}(\nu, \tau)$

using the calculated synchrotron intensity variation (108). 


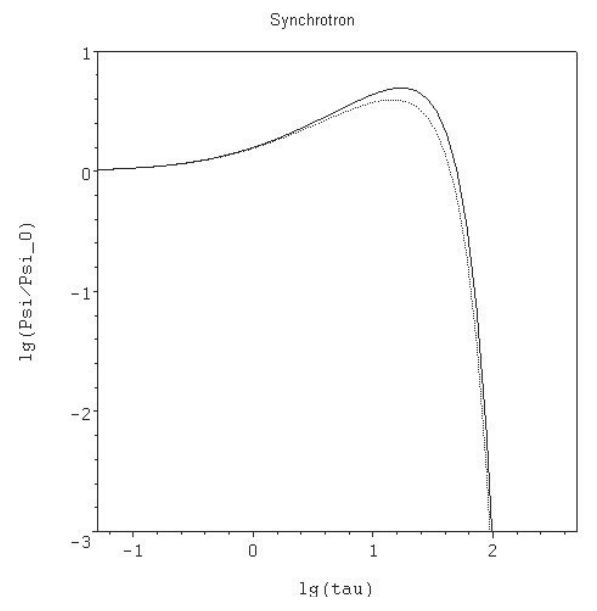

Fig. 3. Emergent synchrotron intensity at $\omega=10^{-3}\left(1.7 b \gamma_{7}^{2} \mathrm{keV}\right)$ in the linear cooling case with (lower curve) and without (upper curve) photon diffusion.

For the specific escape time distributions (135) and (140) we obtain for the synchrotron fluence distribution (77)

$$
\begin{aligned}
f_{s}(v) & =\int_{0}^{\infty} \Psi_{s}(v, \tau) \\
& =\int_{0}^{\tau_{1}} I_{s}(v, \tau) P_{1}(\tau)+\int_{\tau_{1}}^{\infty} I_{s}(\nu, \tau) P_{2}(\tau)
\end{aligned}
$$

which incorporates the effects of spatial photon diffusion in the emission knot.

\subsection{Monochromatic synchrotron light curves}

With an application to observed X-ray light curves we consider the optically thin synchrotron intensity (108)

$I\left(\omega>\omega_{t}, \tau\right)=I_{0} \omega^{1 / 3}(1+\tau)^{2 / 3} \exp \left[-\omega(1+\tau)^{2}\right]$

which increases proportional to $(1+\tau)^{2 / 3}$ for times less than $\tau \leq \omega^{-1 / 2}$ and decays with the Gaussian behaviour $\propto \exp \left(-\omega \tau^{2}\right)$ at large times.

For normalised synchrotron frequencies $\omega>\beta^{2}=$ $1.32 \times 10^{-6} b^{-4} \gamma_{7}^{-2} N_{e, 10}^{-2} R_{15}^{-4}$ the emergent synchrotron intensity is given by

$\psi_{s}(\omega, \tau) \simeq P_{1}(\tau) I\left(\omega>\omega_{t}, \tau\right)=$

$$
0.226 N_{e, 10} R_{15} I_{0} \omega^{1 / 3} \frac{(1+\tau)^{2 / 3}}{(1+\alpha \tau)^{1 / 2}} \exp \left[-\omega(1+\tau)^{2}\right]
$$

which is sketched in Fig. 3. As can be seen, there is no strong influence of the synchrotron photon escape distribution on the light curve of the emergent synchrotron photons. For $\alpha<\omega<1$ there is no influence of the photon escape time distribution and the synchrotron light curve is solely determined by the cooling behaviour of the radiating electrons resulting in the variation (80). For frequencies

$\beta^{2}<\omega<\alpha=3.64 \times 10^{-2} \frac{N_{e, 10}}{b^{2} \gamma_{7}}$

we find the modified increase

$\propto \frac{(1+\tau)^{2 / 3}}{(1+\alpha \tau)^{1 / 2}}$

below the light curve maximum at $\omega^{-1 / 2}$.

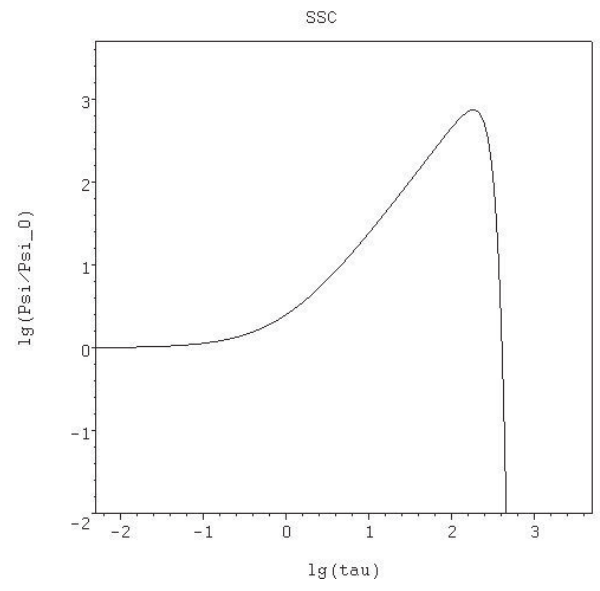

Fig. 4. Emergent monochromatic SSC intensity at $\epsilon_{s}=10^{5}(0.05 \mathrm{TeV})$ in the linear cooling case.

\subsection{Monochromatic and broadband SSC light curves}

At normalised scattered photon energies larger than $\epsilon_{k}(0)$ we use the monochromatic SSC intensity (38)

$\psi_{c}\left(\epsilon_{s}, \tau\right)=4 D_{0} F_{0} P_{0} H\left[\frac{\gamma_{0}}{\epsilon_{s}}-1-\tau\right]\left(\frac{\epsilon_{s}}{\epsilon_{0} \gamma_{0}^{2}}\right)^{1 / 3}$

$\times(1+\tau)^{4 / 3} \exp \left[-\frac{\epsilon_{s} \tau^{4}}{\epsilon_{0} \gamma_{0}^{2}}\right]$

which is sketched in Fig. 4.

Being unaffected by spatial photon diffusion, the emergent SSC light curve is determined by the temporal variations of the relativistic electron density distribution and the synchrotron photon density.

The Heaviside function has a strong influence at high scattered photon energies as it restricts the values of $\tau$ to

$\tau \leq \tau_{T}=\frac{\gamma_{0}}{\epsilon_{s}}-1=100 \frac{\gamma_{7}}{\epsilon_{5}}-1$

where we scaled the intrinsic normalised scattered photon energy at $50 \mathrm{GeV}$, so that $\epsilon_{s}=10^{5} \epsilon_{5}$. The maximum of the SSC intensity (83) as a function of $\tau$ occurs at

$\tau_{m}=\left(\frac{\epsilon_{0} \gamma_{0}^{2}}{3 \epsilon_{s}}\right)^{1 / 4}=\left(\frac{1.13 \times 10^{14} b \gamma_{7}^{4}}{\epsilon_{s}}\right)^{1 / 4}=183\left(\frac{\gamma_{7}}{\epsilon_{5}}\right)^{1 / 4}$

but is only relevant if $\tau_{m}<\tau_{T}$.

Unfortunately, the HESS collaboration due to the lack of enough photons has not published sofar monochromatic TeV light curves from flaring blazars. To have enough photon statistics they have published broad-band TeV light curves: in the case of PKS 2155-304 they give the light curves of all photons with energies greater than $0.2 \mathrm{TeV}$ (see Fig. 1 in Aharonian et al. 2007). We therefore consider broad-band SSC light curves by integrating over the relevant SSC photon energies:

$L\left(\epsilon_{s}, \tau\right)=\int_{\epsilon_{s}}^{\infty} \psi_{c}\left(\epsilon_{s}^{\prime}, \tau\right) \mathrm{d} \epsilon_{s}^{\prime}=\int_{\epsilon_{s}}^{\infty} I_{c}\left(\epsilon_{s}^{\prime}, \tau\right) \mathrm{d} \epsilon_{s}^{\prime}$ 


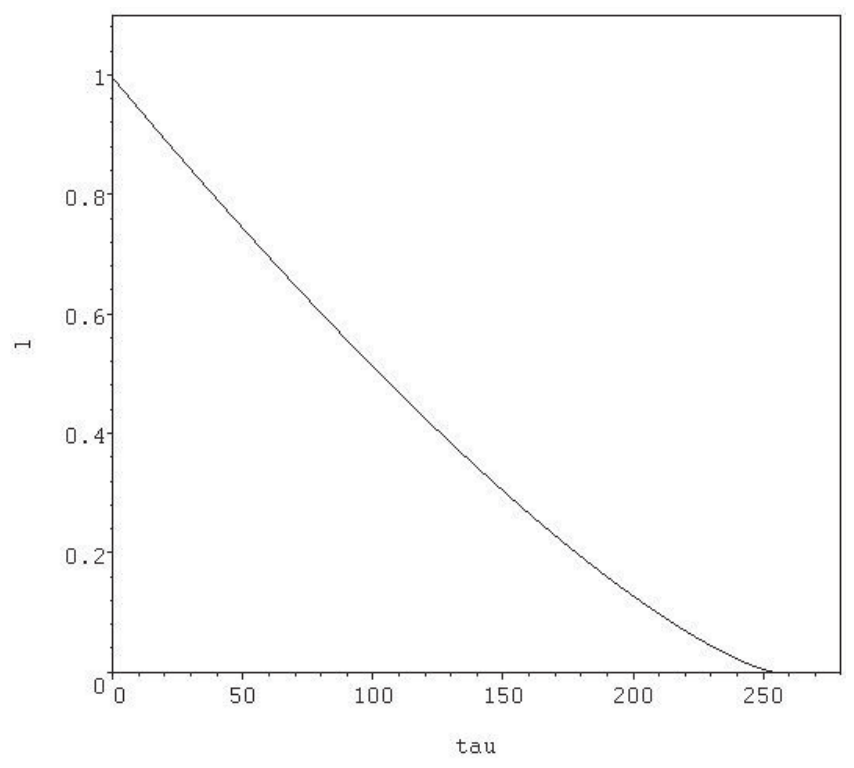

Fig. 5. Broadband SSC light curve $L$ calculated for $E_{\gamma}^{*}=0.2 \mathrm{TeV}$, $\delta_{10}=1$ and $\gamma_{7}=1$.

The SSC intensity (83) readily yields

$$
\begin{aligned}
& L\left(\epsilon_{s}, \tau\right)=\frac{\sigma_{T} R q_{0} I_{0}}{3 \pi}(1+\tau)^{4 / 3} \\
& \quad \times \int_{\epsilon_{s}}^{\infty} \mathrm{d} \epsilon_{s}^{\prime} H\left[\frac{\gamma_{0}}{1+\tau}-\epsilon_{s}^{\prime}\right]\left(\frac{\epsilon_{s}^{\prime}}{\epsilon_{0} \gamma_{0}^{2}}\right)^{1 / 3} \exp \left[-\frac{\epsilon_{s}^{\prime}(1+\tau)^{4}}{\epsilon_{0} \gamma_{0}^{2}}\right] \\
& \quad=4 D_{0} F_{0} \epsilon_{0} \gamma_{0}^{2}(1+\tau)^{-4} H\left[\frac{\gamma_{0}}{1+\tau}-\epsilon_{s}\right] \int_{\frac{\epsilon_{s}(1+\tau)^{4}}{\epsilon_{0} \gamma_{0}^{2}}}^{\frac{(1+\tau)^{3}}{\sigma_{\gamma}}} \mathrm{d} x x^{1 / 3} \mathrm{e}^{-x} \\
& \quad=4 D_{0} F_{0} \epsilon_{0} \gamma_{0}^{2}(1+\tau)^{-4} H\left[\frac{\gamma_{0}}{1+\tau}-\epsilon_{s}\right] \\
& \quad \times\left(\Gamma\left[\frac{4}{3}, \frac{\epsilon_{s}(1+\tau)^{4}}{\epsilon_{0} \gamma_{0}^{2}}\right]-\Gamma\left[\frac{4}{3}, \frac{(1+\tau)^{3}}{\epsilon_{0} \gamma_{0}}\right]\right) .
\end{aligned}
$$

The Heaviside function limits the values of $\tau$ to

$$
1+\tau \leq 255.5 \frac{\gamma_{7} \delta_{10}}{\left(E_{\gamma}^{*} / 0.2 \mathrm{TeV}\right)} \text {. }
$$

On the other hand, the upper integration limit of the $x$-integration in Eq. (87) is less than unity for values of

$$
1+\tau<324 b^{1 / 3} \gamma_{7}
$$

Therefore, for

$$
\left(E_{\gamma}^{*} / 0.2 \mathrm{TeV}\right) b^{1 / 3} \delta_{10}^{-1}>0.79
$$

independent of $\gamma_{7}$, we may approximate Eq. (87) by

$$
\begin{aligned}
L\left(\epsilon_{s}, \tau\right) & \simeq 3 D_{0} F_{0} \frac{\gamma_{0}^{2 / 3}}{\epsilon_{0}^{1 / 3}}\left[1-\left(\frac{\epsilon_{s}(1+\tau)}{\gamma_{0}}\right)^{4 / 3}\right] \\
& =3 D_{0} F_{0} P(\tau) \epsilon_{f}\left[1-\left(\frac{\epsilon_{s}(1+\tau)}{\gamma_{0}}\right)^{4 / 3}\right] .
\end{aligned}
$$

In Fig. 5 we show the time variation of $L\left(\epsilon_{s}, \tau\right)$ for the values $E_{\gamma}^{*}=0.2 \mathrm{TeV}, \delta_{10}=1$ and $\gamma_{7}=1$. This broadband SSC light curve attains its maximum at $\tau=0$ as all photons with energies larger than $\epsilon_{s}$ are counted.

\section{Summary and conclusions}

The vast improvement of sensitivity of modern ground-based air Cherenkov telescopes together with the sensitive flux measurements at lower frequencies requires accurate elaborations of the theoretical radiation models for flaring blazars. Before the appropriate Lorentz transformations of energy and time to the observer's frame of reference, the emergent intrinsic intensities from a relativistic moving emission knot have to be calculated by the product of energy-independent photon escape time distributions $P(T)$ and the energy-dependent radiation intensities $I(\epsilon, t)$ whose time-dependence results from the rapidly cooling energy spectra of the radiating relativistic particles.

Here we consider the flaring of $\mathrm{TeV}$ blazars due to the synchrotron-self Compton (SSC) process. We assume that at the moment $t=t_{0}$ a flare in the emission knot occurs due to the instantaneous injection of monoenergetic $\left(E_{0}\right)$ ultrarelativistic electrons. The ultrarelativistic electrons are injected uniformly over the knot volume and at later times are subject to linear synchrotron radiation cooling in a magnetic field whose strength $B=1 b$ Gauss remains constant during the time evolution of the relativistic electrons. The generated synchrotron photons with nonrelativistic energies are subject to multiple Thomson scattering off the cold electrons in the source giving rise to spatial photon diffusion. Although other (than monoenergetic) injection energy spectra of ultrarelativistic electrons and other (than instantaneous) injection time profiles are physically plausible, the case of monoenergetic instantaneous injection considered here is mathematically very convenient, as the solution of the timedependent kinetic equation for the electrons for this case serves as Green's function for more complex injection energy spectra and time profiles, as long as the electron synchrotron cooling is linear. The solutions for more complex injection energy spectra and time profiles $Q(E, t)$ are then simply obtained by quadratures involving the Green's function solution calculated here. Optically thick and synchrotron radiation intensities and synchrotron photon density distributions in the emission knot as a function of frequency and time are determined. The synchrotron photons serve as target photons for the SSC process which is calculated in the optically thin frequency range using the Thomson approximation of the inverse Compton cross section. It is shown that the optically thick part of the synchrotron radiation process provides a negligible contribution to the resulting SSC intensity at all frequencies and times.

Because the high-energy TeV photons do not undergo multiple elastic Compton scatterings, there is no influence of photon diffusion in the calculation of the SSC fluence distribution with energy. The SSC fluence exhibits a break at $E_{f}=15.8 b^{-1 / 3} \mathrm{GeV}$ from a $\propto E_{s}^{-1 / 4}$-power law spectrum at lower photon energies $E_{t} \leq E_{s} \leq E_{f}$ to a $\propto E_{s}^{-2}\left[1-\left(E_{s} / E_{0}\right)^{7 / 3}\right]$-distribution at large energies $E_{f} \leq E_{s} \leq E_{0}$. The lowest energy limit $E_{t}$ is provided by the synchrotron radiation thickness. The identification of this break energy in the observed fluence distribution from flaring blazars will thus provide diagnostics for the product $\delta b^{-1 / 3}$ of the Doppler factor $\delta$ of the emission knot and the magnetic field strength $b$ in the source. In the case of the observed TeV fluence spectrum of the flare of PKS 2155-304 on July 28, 2006 (Aharonian et al. 2007) we derive $\delta b^{-1 / 3}=27.1 \pm 6.5$.

The emergent SSC light curve is independent of spatial photon diffusion and determined by the temporal variations of the relativistic electron density distribution and the synchrotron photon density. The comparison of the observed with the theoretical monochromatic synchrotron light curve provides the determination of the photon escape distribution. 
Acknowledgements. This work was partially supported by the German Ministry for Education and Research (BMBF) through Verbundforschung Astroteilchenphysik grant $05 \mathrm{CH} 5 \mathrm{PC} 1 / 6$ and the Deutsche Forschungsgemeinschaft through grant Schl 201/16-2.

\section{Appendix A: synchrotron optical depth and photon spectra}

\subsection{Optical depth}

According to Eq. (7) the synchrotron emission is optically thin for frequencies and times with $D(\omega, \tau) \leq 1$, and it is optically thick for frequencies and times with $D(\omega, \tau)>1$, where the transition occurs at the frequency $\omega_{t}(\tau)$ defined by $D\left(\omega_{t}, \tau\right)=1$.

Because $\omega<1$ the optical depth (22) simplifies to

$D(\omega, \tau<1) \simeq\left(\frac{(1+\tau) \omega_{1}}{\omega}\right)^{5 / 3}$

as long as $\omega(1+\tau)^{2}<1$, which holds at normalised times smaller than

$\tau \leq \omega^{-1 / 2}-1 \simeq \omega^{-1 / 2}$.

Consequently, in this range the transition frequency is given by

$\omega_{t}\left(\tau<\omega^{-1 / 2}\right) \simeq \omega_{1}(1+\tau)$.

It is instructive to inspect the limit $\tau>1$ in more detail. Here the optical depth (22) reduces to

$D(\omega, \tau>1) \simeq\left(\tau \frac{\omega_{1}}{\omega}\right)^{5 / 3}\left[1+\frac{3}{2} \omega \tau^{2}\right] \exp \left[-\omega \tau^{2}\right]$.

In terms of the combined variable $z=\omega \tau^{2}$ this reads

$D(z)=\omega_{1}^{5 / 3} \tau^{5} f(z), \quad f(z)=\frac{1+1.5 z}{z^{5 / 3}} \mathrm{e}^{-z}$.

The function $f(z)$ is a monotonically decreasing function for all values of $z$ with the asymptotic behaviour

$f(z) \simeq \begin{cases}z^{-5 / 3} & \text { for } z \leq 1 \\ 1.5 z^{-2 / 3} \mathrm{e}^{-z} & \text { for } z>1\end{cases}$

For values $z<1$, corresponding to the case (93) considered before, use of the asymptotic behaviour immediately yields

$D(z) \simeq \omega_{1}^{5 / 3} \tau^{5} z^{-5 / 3}=\left(\frac{\tau \omega_{1}}{\omega}\right)^{5 / 3}$

and the transition frequency $\omega_{t} \simeq \tau \omega_{1}$ in agreement with the result (94).

At the value $z=1$ the function $f(1)=0.92$ is already smaller than unity. Hence, the equation defining the optical transition frequency $\left(z_{t}=\tau^{2} \omega_{t}\right)$ in the range of large values of $z>1$ is

$D\left(z_{t}\right)=1=\omega_{1}^{5 / 3} \tau^{5} f\left(z_{t}\right)<0.92 \omega_{1}^{5 / 3} \tau^{5}$.

This equation can be solved for $z_{t}>1$ only if

$0.92 \omega_{1}^{5 / 3} \tau^{5}>1$

corresponding to

$\tau>\omega_{1}^{-1 / 3}$.

In the opposite case $\tau<\omega_{1}^{-1 / 3}$ the function $D\left(z_{t}\right)$ is always smaller than unity for all values of $z_{t}>1$ so that the transition

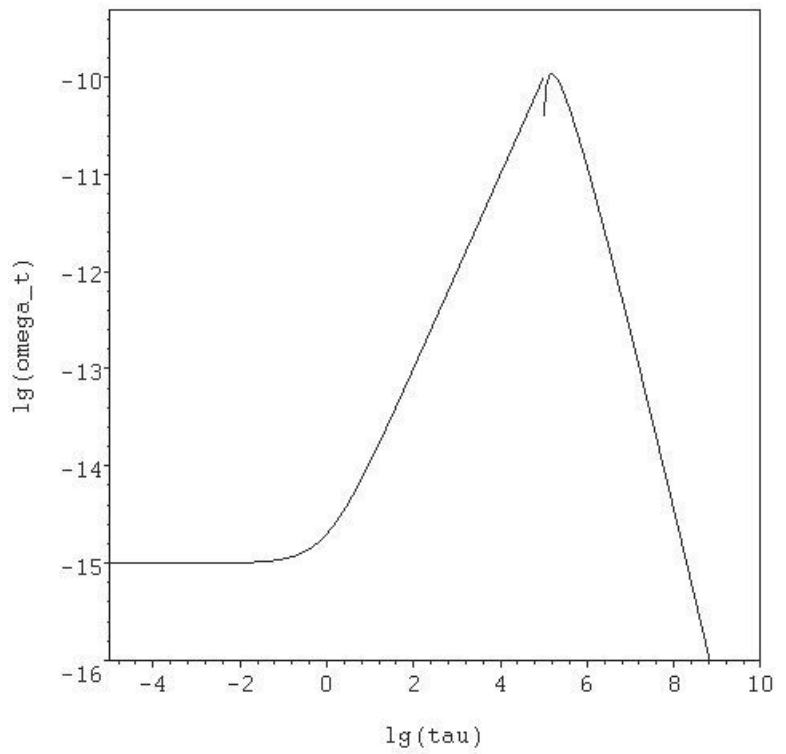

Fig. 6. Normalised synchrotron transition frequency $\omega_{t}$ as a function of the normalised time scale $\tau$ in the linear cooling case calculated for $\omega_{1}=10^{-15}$. The maximum transition frequency $\omega_{1}^{2 / 3}=10^{-10}$ occurs at the time $\tau=\omega_{1}^{-1 / 3}=10^{5}$.

frequency in this case has to be in the range $z_{t}<1$, discussed before (see Eq. (98)).

With the asymptotic expansion (97) we find the solution $z_{t}>$ 1 from the transcendental equation

$z_{t}^{2 / 3} \mathrm{e}^{z_{t}}=1.5 \omega_{1}^{5 / 3} \tau^{5}$

yielding approximately

$z_{t} \simeq \ln \left(1.5 \omega_{1}^{5 / 3} \tau^{5}\right)$

corresponding to

$\omega_{t} \simeq \frac{1}{\tau^{2}} \ln \left(1.5 \omega_{1}^{5 / 3} \tau^{5}\right)$

Summarizing this subsection: for the time-dependence of the transition frequency we derive

$\omega_{t}(\tau) \simeq \begin{cases}\omega_{1}(1+\tau) & \text { for } \tau \leq \omega_{1}^{-1 / 3} \\ \frac{1}{\tau^{2}} \ln \left(1.5 \omega_{1}^{5 / 3} \tau^{5}\right) & \text { for } \tau>\omega_{1}^{-1 / 3}\end{cases}$

which is sketched in Fig. 6. After the constant value $\omega_{1}$ at small normalised times $\tau<1$ the transition frequency increases linearly to its maximum value $\omega_{1}^{2 / 3}$ at $\tau=\omega_{1}^{-1 / 3}$. At late times $\tau>\omega_{1}^{-1 / 3}$ the transition frequency decreases proportional to $\tau^{-2}$.

In the two normalised time ranges the optical thickness can be well approximated as

$D(\omega, \tau) \simeq$

$\begin{cases}\left(\frac{(1+\tau) \omega_{1}}{\omega}\right)^{5 / 3} & \text { for } 0 \leq \tau \leq \omega_{1}^{-1 / 3} \\ \left(\frac{\omega_{t}}{\omega}\right)^{2 / 3} \exp \left(\tau^{2}\left(\omega_{t}-\omega\right)\right) & \text { for } \tau>\omega_{1}^{-1 / 3}\end{cases}$ 


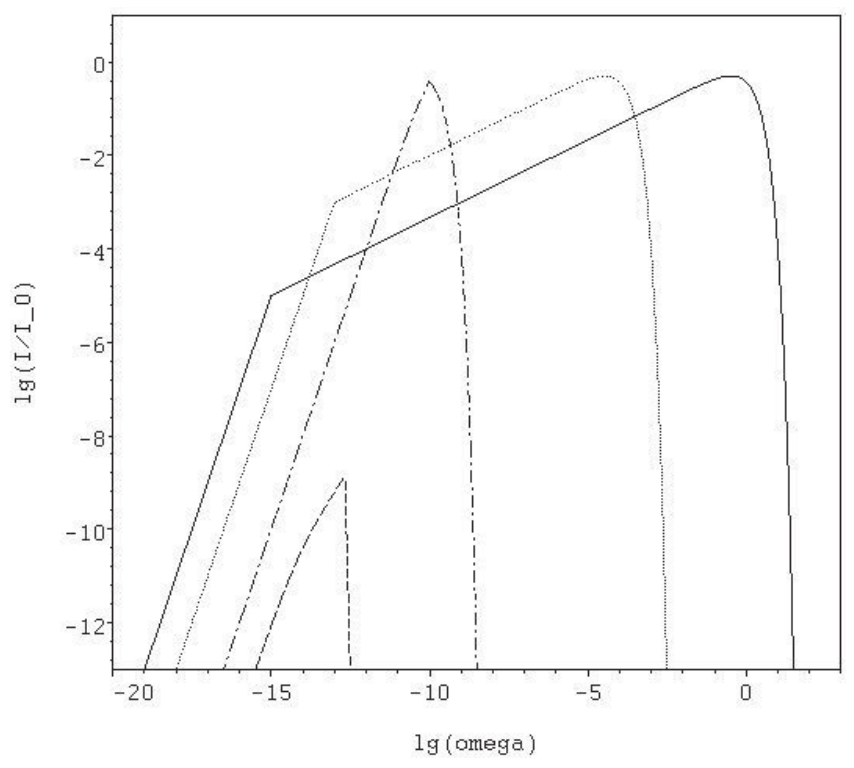

Fig. 7. Synchrotron intensity distribution as a function of the normalised frequency $\omega$ at different times $\tau=0$ (full curve), $\tau=100$ (dotted curve), $\tau=10^{5}$ (dot-dashed curve) and $\tau=10^{7}$ (dashed curve). The value $\omega_{1}=10^{-15}$ is adopted.

\subsection{Synchrotron spectra}

In the optically thick frequency part $\omega \leq \omega_{t}$ the synchrotron intensity according to Eq. (7) is given by

$$
\begin{aligned}
I\left(\omega \leq \omega_{t}, \tau\right) & \simeq \frac{j(\omega, \tau) R}{D(\omega, \tau)}=\frac{3 m v_{s}^{2} \gamma_{0}^{5} \omega^{2}}{2(1+\tau)\left[1+\frac{3}{2} \omega(1+\tau)^{2}\right]} \\
& =I_{0} \omega_{1}^{-5 / 3} \frac{\omega^{2}}{(1+\tau)\left[1+\frac{3}{2} \omega(1+\tau)^{2}\right]}
\end{aligned}
$$

where

$$
\begin{aligned}
I_{0} & =\frac{3}{2} m v_{s}^{2} \gamma_{0}^{5} \omega_{1}^{5 / 3}=a_{0} P_{0} q_{0} R v_{s} /(4 \pi) \\
& =3.8 \times 10^{-3} q_{5} R_{15} b \quad \frac{\mathrm{erg}}{\mathrm{cm}^{2} \text { ster Hz s}}
\end{aligned}
$$

is determined by the magnetic field strength $b$, the electron injection rate $q_{5}$ and the source radius $R_{15}$.

In the optically thin frequency range we obtain

$$
\begin{aligned}
I\left(\omega>\omega_{t}, \tau\right) & \simeq j(\omega, \tau) R \\
& =I_{0}\left[\omega(1+\tau)^{2}\right]^{1 / 3} \exp \left[-\omega(1+\tau)^{2}\right] .
\end{aligned}
$$

In Fig. 7 we show the intensity distribution as a function of frequency at different times.

In each case the intensity distribution is characterised by two intensities:

(i) the time-independent peak intensity

$I_{m}=\frac{I_{0}}{(3 e)^{1 / 3}}=0.497 I_{0}$

occurring in the optically thin frequency part at

$\omega_{m}(\tau)=\frac{1}{3}(1+\tau)^{-2}$,

and

(ii) the intensity $I_{\text {trans }}(\tau)=I\left(\omega_{t}, \tau\right)$ at the transition frequency $\omega_{t}$, hereafter referred to as transition intensity.

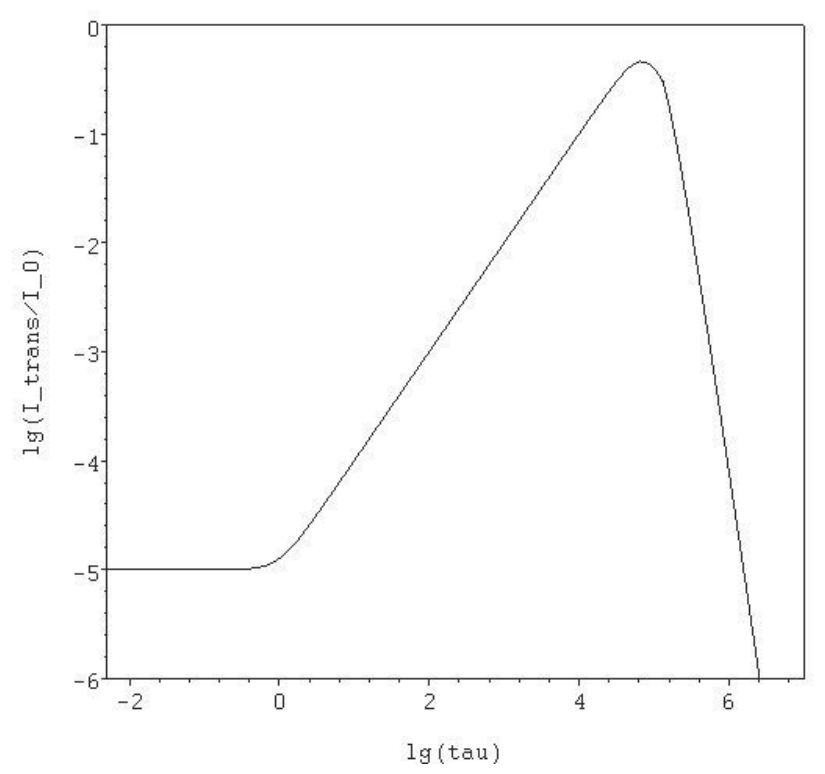

Fig. 8. Synchrotron transition intensity $I_{\text {trans }}(\tau) / I_{0}$ as a function of the normalised time scale calculated for $\omega_{1}=10^{-15}$.

\subsubsection{Transition intensity}

The transition intensity is given by

$$
\begin{aligned}
I_{\text {trans }}(\tau) & =I\left(\omega_{t}, \tau\right) \\
& =I_{0} \omega_{1}^{-5 / 3} \frac{\omega_{t}^{2}}{(1+\tau)\left[1+\frac{3}{2} \omega_{t}(1+\tau)^{2}\right]}
\end{aligned}
$$

Using the transition frequencies (104) we obtain for the transition intensity at normalised times smaller than $\omega_{1}^{-1 / 3}$

$$
I_{\text {trans }}\left(\tau \leq \omega_{1}^{-1 / 3}\right) \simeq I_{0} \frac{\left[\omega_{1}(1+\tau)^{3}\right]^{1 / 3}}{1+\frac{3}{2} \omega_{1}(1+\tau)^{3}}
$$

whereas at large times

$I_{\text {trans }}\left(\tau>\omega_{1}^{-1 / 3}\right) \simeq \frac{2 I_{0}}{3} \frac{\omega_{t}}{\tau^{3} \omega_{1}^{5 / 3}}=\frac{2 I_{0}}{3} \frac{\ln \left[1.5\left(\omega_{1}^{1 / 3} \tau\right)^{5}\right]}{\left(\omega_{1}^{1 / 3} \tau\right)^{5}}$

The time-dependence of the transition intensity is shown in Fig. 8.

At small times $\tau \leq 1$ the transition intensity is given by

$$
\begin{aligned}
& I_{\text {trans }}(\tau \leq 1) \simeq I_{0} \omega_{1}^{1 / 3} \\
& \quad=1.15 \times 10^{-7} q_{5}^{6 / 5} R_{15}^{6 / 5} b^{4 / 5} \gamma_{7}^{-1} \frac{\mathrm{erg}}{\mathrm{cm}^{2} \operatorname{ster~Hz~s}}
\end{aligned}
$$

at intermediate times $1<\tau \leq \omega_{1}^{-1 / 3}$ the transition intensity increases linearly with time to its maximum value

$I_{\text {trans, } \max }=I_{\text {trans }}\left(\omega_{1}^{-1 / 3}\right)=0.4 I_{0}$.

Note that the value (115) is indeed smaller than the peak intensity (109). At large times $\tau>\omega_{1}^{-1 / 3}$ the transition intensity rapidly decreases $\propto \tau^{-5}$. 


\subsubsection{Peak intensity}

The peak intensity (109) has a value independent of time but occurs at the frequency $\omega_{m}$, (see Eq. (110)), which decreases $\propto \tau^{-2}$ at times larger than unity. Comparing with the time variation (104) of the transition frequency we conclude that only at times smaller $\tau<\omega_{1}^{-1 / 3}$ the frequency $\omega_{m}>\omega_{t}$ is larger than the transition frequency. Consequently, broad optically thin synchrotron emission only occurs for times less than $\omega_{1}^{-1 / 3}$, as clearly seen in Fig. 7. At large times $\tau>\omega_{1}^{-1 / 3}$, the synchrotron intensity is much suppressed with the maximum intensity given by the transition intensity (113). This behaviour is also seen in Fig. 7.

\section{Appendix B: Photon escape time distribution}

Here we calculate the distribution of photons $P(v, t)$ over their time of escape from the emission knot (Sunyaev \& Titarchuk 1980, 1985). The optical depth $K_{0}=\sigma_{T} N_{e} R=6.65 N_{e, 10} R_{15}$ of the knot with respect to Thomson scattering off the cold electrons in the knot of density $N_{e}=10^{10} N_{e, 10} \mathrm{~cm}^{-3}$ results in the spatial diffusion of photons in the emission knot after their generation. The average photon intensity $J(r, t)$ in the knot $0 \leq r \leq R$ obeys the diffusion equation (Sunyaev \& Titarchuk 1980; Rybicki \& Lightman 1979)

$\frac{\partial J}{\partial t}=\frac{c}{3 \sigma_{T} N_{e}} \frac{1}{r^{2}} \frac{\partial}{\partial r}\left(r^{2} \frac{\partial J}{\partial r}\right)$.

Introducing the Thomson optical depth $K=\sigma_{T} N_{e} r$ and the dimensionless scattering time $u=c \sigma_{T} N_{e}\left(t-t_{0}\right)$ yields for Eq. (116)

$\frac{\partial J}{\partial u}=\frac{1}{3} \frac{1}{K^{2}} \frac{\partial}{\partial K}\left(K^{2} \frac{\partial J}{\partial K}\right)$

which has to be solved for the boundary conditions that $J(0, t)$ is finite at the centre of the knot and that at $r=R$ there is no photon flux from outside onto the boundary surface of the spherical knot:

$\left[\frac{\partial J}{\partial K}+\frac{3}{2} J\right]_{K=K_{0}}=0$.

The initial condition

$$
J(K, u=0)=H\left[K_{0}-K\right]
$$

reflects that at the time $t_{0}$ synchrotron and SSC photons are generated uniformly over the emission knots by the injected ultrarelativistic electrons. From the solution $J\left(K_{0}, u\right)$ the normalised photon escape time distribution is calculated as

$P\left(K_{0}, u\right)=\frac{J\left(K_{0}, u\right)}{\int_{0}^{\infty} \mathrm{d} u J_{0}\left(K_{0}, u\right)}$.

In principal, the Thomson scattering off cold electrons also leads to a diffusion of photons in frequency (Comptonization); we neglect this frequency diffusion here because it is slow compared to the synchrotron and SSC interactions with the relativistic electrons.

The solutions of the photon spatial transport are different for small and large values of the Thomson optical depth $K_{0}=$ $6.65 N_{e, 10} R_{15}$. We discuss both cases in turn.

\subsection{Small knot Thomson depth}

At small optical Thomson depth $K_{0} \ll 1$ the distribution of multiply scattered photons over the knot will be uniform. Therefore the part of the photons escaping from the knot depends only on the Thomson depth and is given by (Sunyaev \& Titarchuk 1985)

$P\left(K_{0} \ll 1, u\right)=\frac{\exp \left[-u \ln \left(\frac{4}{3 K_{0}}\right)\right]}{\ln \left(\frac{4}{3 K_{0}}\right)}$.

This estimate has been verified in numerical Monte Carlo calculations (Pozdnyakov et al. 1976).

\subsection{Large knot Thomson depth}

The solution of the photon diffusion problem (117)-(119) is well known (Sunyaev \& Titarchuk 1980). Because of several misprints and missing details we repeat the derivation here. The solution of Eq. (117) is

$J(K, u)=\sum_{n=1}^{\infty} c_{n} \frac{\sin \left(\lambda_{n} K\right)}{K} \mathrm{e}^{-\lambda_{n}^{2} u / 3}$

where the eigenvalues $\lambda_{n}$ fulfil the transcendental equation

$\frac{\tan \left(K_{0} \lambda_{n}\right)}{K_{0} \lambda_{n}}=\frac{1}{1-\frac{3 K_{0}}{2}}$

and the expansion coefficients $c_{n}$ are determined by the initial condition (119) as

$$
\begin{aligned}
c_{n} & =\frac{1}{\lambda_{n}} \frac{\int_{0}^{K_{0} \lambda_{n}} \mathrm{~d} x x \sin x}{\int_{0}^{K_{0} \lambda_{n}} \mathrm{~d} x \sin ^{2} x} \\
& =\frac{2}{\lambda_{n}} \frac{\sin \left(K_{0} \lambda_{n}\right)-K_{0} \lambda_{n} \cos \left(K_{0} \lambda_{n}\right)}{K_{0} \lambda_{n}-\sin \left(K_{0} \lambda_{n}\right) \cos \left(K_{0} \lambda_{n}\right)}
\end{aligned}
$$

The intensity at the knot's edge then is

$J\left(K_{0}, u\right)=\sum_{n=1}^{\infty} F\left(K_{0} \lambda_{n}\right) \mathrm{e}^{-\lambda_{n}^{2} u / 3}$

where

$F(y)=\frac{2 \sin y}{y} \frac{\sin y-y \cos y}{y-\sin y \cos y}$

Inserting the eigenvalue equation (123) in the form $\tan y=$ $y\left(1-\frac{3}{2} K_{0}\right)^{-1}$ yields

$F(y)=\frac{3 K_{0}}{y^{2}-\frac{3}{2} K_{0}+\frac{9}{4} K_{0}^{2}}$

so that

$F\left(K_{0} \lambda_{n}\right)=\frac{4}{3 K_{0}} \frac{1}{1-\frac{2}{3 K_{0}}+\frac{4}{9} \lambda_{n}^{2}}$.

Equation (125) then becomes

$J\left(K_{0}, u\right)=\frac{4}{3 K_{0}} \sum_{n=1}^{\infty} \frac{\mathrm{e}^{-\lambda_{n}^{2} u / 3}}{1-\frac{2}{3 K_{0}}+\frac{4}{9} \lambda_{n}^{2}}$ 
which for $K_{0} \gg 1$ agrees with the distribution given by Sunyaev \& Titarchuk (1980, Appendix A):

$J\left(K_{0} \gg 1, u\right) \simeq \frac{4}{3 K_{0}} \sum_{n=1}^{\infty} \frac{\mathrm{e}^{-\lambda_{n}^{2} u / 3}}{1+\frac{4}{9} \lambda_{n}^{2}}$.

For the escape time distribution we find

$P(u)=\frac{3}{2 K_{0}} J\left(K_{0} \gg 1, u\right)=\frac{2}{K_{0}^{2}} \sum_{n=1}^{\infty} \frac{\mathrm{e}^{-\lambda_{n}^{2} u / 3}}{1+\frac{4}{9} \lambda_{n}^{2}}$.

For large Thomson depth $K_{0} \gg 1$ the transcendental eigenvalue Eq. (123) is

$\frac{\tan \left(K_{0} \lambda_{n}\right)}{K_{0} \lambda_{n}} \simeq-\frac{2}{3 K_{0}} \simeq 0$

yielding the eigenvalues

$\lambda_{n} \simeq \frac{n \pi}{K_{0}}$

so that

$P(u) \simeq \frac{2}{K_{0}^{2}} \sum_{n=1}^{\infty} \frac{\mathrm{e}^{-\frac{n^{2} \pi^{2} u}{3 K_{0}^{2}}}}{1+\left(\frac{2 n \pi}{3 K_{0}}\right)^{2}}$

with the following asymptotic behaviour.

For large scattering times $u \gg K_{0}^{2} \gg 1$ the lowest eigenvalue provides the exponential cut-off, so that

$P_{2}\left(u \geq K_{0}^{2}\right) \simeq \frac{2}{K_{0}^{2}} \mathrm{e}^{-\frac{\pi^{2} u}{3 K_{0}^{2}}}$.

For times $u \leq K_{0}^{2}$ many $n$ contribute to the sum in Eq. (134), so that $n$ is treated as a continuous variable:

$P\left(u \leq K_{0}^{2}\right)=\frac{2}{K_{0}^{2}}\left[\int_{0}^{\infty} \mathrm{d} n \frac{\mathrm{e}^{-\frac{n^{2} \pi^{2} u}{3 K_{0}^{2}}}}{1+\left(\frac{2 n \pi}{3 K_{0}}\right)^{2}}-1\right]$.

The substitution $n=3 K_{0} t /(2 \pi)$ yields

$$
\begin{aligned}
P\left(u \leq K_{0}^{2}\right) & =\frac{2}{K_{0}^{2}}\left[\frac{3 K_{0}}{2 \pi} \int_{0}^{\infty} \mathrm{d} t \frac{\mathrm{e}^{-\frac{3 u}{4} t^{2}}}{1+t^{2}}-1\right] \\
& =\frac{2}{K_{0}^{2}}\left[\frac{3 K_{0}}{4} \mathrm{e}^{\frac{3 u}{4}} \operatorname{erfc}\left(\sqrt{\frac{3 u}{4}}\right)-1\right]
\end{aligned}
$$

in terms of the complementary error function (Abramowitz \& Stegun 1972).

For intermediate scattering times $1 \leq u \leq K_{0}^{2}$ use of

$\mathrm{e}^{z^{2}} \operatorname{erfc}(z) \simeq \frac{1}{\pi^{1 / 2} z}\left(1-\frac{1}{2 z^{2}}\right)$

yields

$$
\begin{aligned}
P\left(1 \leq u \leq K_{0}^{2}\right) & \simeq \frac{2}{K_{0}^{2}}\left[\frac{3 K_{0}}{2} \frac{1}{\sqrt{3 \pi u}}\left(1-\frac{2}{3 u}\right)\right] \\
& =\sqrt{\frac{3}{\pi}} \frac{1}{K_{0} u^{1 / 2}}\left(1-\frac{2}{3 u}\right) .
\end{aligned}
$$

At small times $u<1$ we obtain approximately

$P(u<1) \simeq \frac{3}{2 K_{0}}\left[1-\sqrt{\frac{3 u}{\pi}}\right]$.

The two approximations (138) and (139) are reasonablely approximated by the combined function

$P_{1}\left(u \leq K_{0}^{2}\right) \simeq \frac{3}{2 K_{0}}\left[1+\frac{3 \pi u}{4}\right]^{-1 / 2}$.

For large optical Thomson depth $K_{0} \gg 1$ the escape distributions (135) and (140) yield for the average photon escape time after elementary integrations

$$
\begin{aligned}
\langle t\rangle= & \frac{1}{c \sigma_{T} N_{e}} \int_{0}^{\infty} \mathrm{d} u u P(u) \\
& =\frac{R}{c K_{0}}\left(\int_{0}^{K_{0}^{2}} \mathrm{~d} u u P_{1}(u)+\int_{K_{0}^{2}}^{\infty} \mathrm{d} u u P_{2}(u)\right) \\
& =\frac{2 R}{c K_{0}^{3}}\left[\int_{K_{0}^{2}}^{\infty} \mathrm{d} u u \exp \left[-\frac{\pi^{2} u}{3 K_{0}^{2}}\right]\right. \\
+ & \left.\frac{3 K_{0}}{4} \int_{0}^{K_{0}^{2}} \mathrm{~d} u u\left[1+\frac{3 \pi u}{4}\right]^{-1 / 2}\right] \\
& =\frac{16 R}{9 \pi^{2} c K_{0}^{2}}\left[\frac{27}{8}\left(1+\frac{3}{\pi^{2}}\right) \mathrm{e}^{-\pi^{2} / 3} K_{0}^{3}+2\right. \\
& \left.+\left(1+\frac{3 \pi K_{0}^{2}}{4}\right)^{3 / 2}-3\left(1+\frac{3 \pi K_{0}^{2}}{4}\right)^{1 / 2}\right] .
\end{aligned}
$$

To leading order in $K_{0} \ll 1$ we obtain

$$
\begin{aligned}
\langle t\rangle & =\frac{2}{3 \pi^{2}} \frac{R K_{0}}{c}\left[9\left(1+\frac{3}{\pi^{2}}\right) \mathrm{e}^{-\pi^{2} / 3}+\pi \sqrt{3 \pi}\right] \\
& =1.53 \frac{R K_{0}}{c}=3.4 \times 10^{5} R_{15}^{2} N_{e, 10} \mathrm{~s} .
\end{aligned}
$$

\section{References}

Abramowitz, M., \& Stegun, I. S. 1972, National Bureau of Standards, Washington

Aharonian, F. A., Aharonian, F., Akhperjanian, A. G., Bazer-Bachi, A. R., et al. (HESS Collaboration) 2007, ApJ, 664, L7

Albert, J., Albert, J., Aliu, E., Anderhub, H., et al. (MAGIC Collaboration) 2007, ApJ, 663, 125

Böttcher, M. 2007, Ap\&SS, 309, 95

Chiaberge, M., \& Ghisellini, G. 1999, MNRAS, 306, 551

Dermer, C. D., \& Schlickeiser, R. 1993, ApJ, 416, 458

Dermer, C. D., Sturner, S. J., \& Schlickeiser, R. 1997, ApJS, 109, 103

Fabian, A. C., Blandfors, R. D., Guilbert, P. W., Phinney, E. S., \& Cuellar, L. 1986, MNRAS, 221, 931

Foschini, L., Ghisellini, G., Tavecchio, F., et al. 2007, ApJ, 657, L81

Frederiksen, J. T., Hededal, C. B., Haugbolle, T., \& Nordlund, A. 2004, ApJ, 608, L13

Gerbig, D., \& Schlickeiser, R. 2000, ApJ, 664, 750 
Jaroschek, C., Lesch, H., \& Treumann, R. 2005, ApJ, 618, 822

Kapetanakos, C. A. 1974, Appl. Phys. Lett., 25, 484

Kardashev, N. S. 1962, Sov. Astron. - AJ, 6, 317

Lee, R., \& Lampe, M. 1973, Phys. Rev. Lett., 31, 1390

Lightman, A. P., \& Zdziarski, A. A. 1987, ApJ, 319, 643

Mastichiadis, A., \& Kirk, J. G. 1997, A\&A, 320, 19

$\mathrm{Ng}$, J. S. T., \& Noble, R. J. 2006, Phys. Rev. Lett., 96, 115006

Nishikawa, K. I., Hardee, P., Richarson, G., et al. 2003, ApJ, 595, 555

Piner, B. G., \& Edwards, P. G. 2004, ApJ, 600, 115

Pohl, M., \& Schlickeiser, R. 2000, A\&A, 354, 395

Pozdnyakov, L. A., Sobol, I. M., \& Sunyaev, R. A. 1976, Sov. Astron. Lett., 2, 55

Rybicki, G. B., \& Lightman, A. P. 1979, Radiative Processes in Astrophysics (New York: Wiley)
Sakai, J.-I., Schlickeiser, R., \& Sukla, P. K. 2004, Phys. Lett. A, 330, 384

Schlickeiser, R. 1996, Space Science Rev., 75, 299

Schlickeiser, R., \& Lerche, I. 2007a, A\&A, 476, 1

Schlickeiser, R., \& Lerche, I. 2007b, A\&A, submitted

Schlickeiser, R., Vainio, R., Böttcher, M., Pohl, M., Lerche. I., \& Schuster, C. 2002, A\&A, 393, 69

Silva, L. O., Fonseca, R. A., Tonge, J. W., et al. 2003, ApJ, 596, L121

Sokolov, A., Marscher, A. P., \& McHardy, I. M. 2004, ApJ, 613, 725

Stockem, A., Lerche, I., \& Schlickeiser, R. 2007, ApJ, 659, 419

Sunyaev, R. A., \& Titarchuk, L. G. 1980, A\&A, 86, 121

Sunyaev, R. A., \& Titarchuk, L. G. 1985, A\&A, 143, 374

Tatarakis, M., et al. 2003, Phys. Rev. Lett., 90, 175001

Woo, J.-H., Woo, Jong-Hak, Urry, C., Megan, van der Marel, Roeland, P., et al. 2005, ApJ, 631, 762 